\title{
On the ability of the spectroscopic Sunyaev-Zeldovich effect measurements to determine the temperature structure of galaxy clusters
}

\author{
S. Colafrancesco ${ }^{1,2}$ and P. Marchegiani ${ }^{2,3}$
}

\author{
1 ASI, V.le Liegi 26, 00100 Roma, Italy \\ e-mail: Sergio.Colafrancesco@asi.it \\ 2 INAF-Osservatorio Astronomico di Roma via Frascati 33, 00040 Monteporzio, Italy \\ e-mail: cola@mporzio.astro.it \\ 3 Dipartimento di Fisica, Università di Roma La Sapienza, P.le A. Moro 2, Roma, Italy \\ e-mail: marchegiani@mporzio.astro.it
}

Received 13 August 2009 / Accepted 14 February 2010

\begin{abstract}
Aims. We explore the ability of spatially resolved spectroscopic measurements of the SZ effect (SZE) to determine the temperature profile of galaxy clusters. We derive a general formalism for the thermal SZE in galaxy clusters with a non-uniform temperature profile that can be applied to both cool-core clusters and non-cool-core clusters with an isothermal or non-isothermal temperature structure.

Methods. We develop an inversion technique by means of which the electron distribution function can be extracted from spectroscopic SZE observations over a wide frequency range. We study the fitting procedure to extract the cluster temperature from a set of simulated spatially resolved spectroscopic SZE observations in different bands of the spectrum from 100 to $450 \mathrm{GHz}$.

Results. We present our analysis results for three different cluster prototypes: A2199 with a low-temperature cool core, Perseus with a relatively high-temperature cool core, and Ophiuchus with an isothermal temperature distribution. These results indicate both the precision of the SZE observations and the optimal frequency bands required to determine the cluster temperature with similar or better accuracy than that obtainable from X-ray observations. The precision of SZE-derived temperature is also discussed for the outer regions of clusters. Using our method, we also study the possibility of extracting the parameters characterizing the non-thermal SZE spectrum of the relativistic plasma contained in the lobes of radio galaxies as well as the spectrum of relativistic electrons cospatially distributed with the thermal plasma in clusters that exhibit non-thermal phenomena.

Conclusions. We find that the next generation SZE experiments, which will have both spectroscopic capabilities with moderate resolution of a few to tens $\mathrm{GHz}$ and imaging capabilities with spatial resolution of tens of arcsec up to arcmin, can provide precise temperature distribution measurements over a wide range of radial distances for galaxy clusters even out to high redshift.
\end{abstract}

Key words. cosmology: theory - galaxies: clusters: general

\section{Introduction}

Galaxy clusters are the largest gravitationally bound container of diffuse baryons in the universe. These baryons accumulate in the cluster gravitational potential, which is dominated by cold dark matter and show, in their hotter phase (i.e. the hot intracluster medium - hereafter ICM - of temperature $\left.T_{\mathrm{e}} \sim 10^{7}-10^{8} \mathrm{~K}\right)$, a complex temperature distribution in the cluster atmospheres as indicated by observations (see, e.g., Arnaud 2005, for a review; Pratt et al. 2007, for XMM-Newton results; Ehlert \& Ulmer 2009, for Chandra results; Sato et al. 2009, for Suzaku results) and numerical simulations (see Borgani et al. 2008, for a review).

Spatially resolved studies of the ICM temperature distribution in galaxy clusters are crucial for both astrophysical and cosmological applications since these studies provide information about the thermodynamic state of the ICM, allowing us to measure the total mass of these systems by means of the hydrostatic equilibrium equation (see e.g. Arnaud 2005; Colafrancesco \& Giordano 2007) and to place constraints on the cosmological parameters $\Omega_{\mathrm{m}}, \Omega_{\mathrm{DE}}, \Omega_{\mathrm{b}}$ by studying the cluster population evolution.
On the cosmological side, once the gas mass is determined from X-ray observations in the deprojection or fitting analysis (see, e.g., Fabian et al. 1981; Ettori \& Fabian 1999) and the total mass is estimated by means of the hydrostatic equilibrium condition, it is possible to derive the cluster gas mass fraction that can be used to constrain the cosmological density parameter, if combined with primordial nucleosynthesis calculations (e.g., White et al. 1993; Ettori et al. 2009). Cosmological applications of the cluster population evolution require precise measurements of the cluster temperature and of the correlation between temperature and other relevant physical quantities, to describe correctly the cluster population (total mass, luminosity, Compton parameter, number counts, redshift distribution, luminosity function). If well calibrated, the slope and evolution of cluster scaling relations, such as gas mass versus temperature (e.g., Colafrancesco \& Vittorio 1994; Colafrancesco et al. 1994, 1997; Voit 2000; Finoguenov et al. 2001) and cluster size versus temperature (e.g., Colafrancesco et al. 1997; Mohr et al. 2000; Verde et al. 2001), can also be used to constrain cosmological and structure formation models. 
On the astrophysical side, the precise determination of important quantities such as entropy, pressure, and - based on the assumption of hydrostatic equilibrium - the total cluster mass, is dependent on an accurate estimation of the temperature profile. Accurate temperature profiles are fundamental in determining the gas entropy distribution (see Lloyd-Davies et al. 2000, and references therein), which is a powerful tool for exploring the role of non-gravitational processes that could alter the specific thermal energy in the ICM (Kang et al. 2007; Colafrancesco \& Giordano 2006, 2007; for a review see Colafrancesco 2008a), assessing the structure of cool cores and their surrounding atmosphere to probe the interplay of non-thermal and thermal particle distributions in clusters (e.g., Colafrancesco et al. 2004; Guo \& Ho 2008; Colafrancesco \& Marchegiani 2008, and references therein), and assessing the amount of non-thermal (pointlike and/or diffuse) emission mechanisms detectable in the hard $\mathrm{X}$-ray domain (see discussion in Colafrancesco \& Marchegiani 2009).

The standard methodology for recovering the ICM temperature distribution uses high resolution spectroscopic X-ray observations (XMM, Chandra, Suzaku and previously ROSAT, ASCA, and BeppoSAX). These observations provide the key measurable characteristics of the ICM, i.e., the temperature and density of this plasma. Because of limited photon statistics, the density and temperature are usually measured in terms of radial profiles (see, e.g., Pratt et al. 2007). However, while the density of the ICM is relatively easy to measure from the surface brightness profile of a galaxy cluster, precise temperature determination requires high photon statistics to build, and fit, a spectrum. Therefore, ICM temperature profiles are typically determined with considerably lower spatial resolution than density profiles. The measurement of radial temperature profiles is complicated by the density squared dependence of the thermal bremsstrahlung X-ray emissivity, $\varepsilon_{\text {brem }} \propto n_{\mathrm{e}}^{2}(r) T_{\mathrm{e}}^{1 / 2}(r)$. The steep drop in the $\mathrm{X}$-ray surface brightness with distance from the centre, combined with the background from cosmic, solar, and instrumental sources, makes, hence, accurate X-ray measurement of the temperature distribution at large distances from the cluster centre a technically challenging task (see discussion in Pratt et al. 2007).

The ICM temperature can alternatively be measured by using the inverse Compton scattering of CMB photons off thermal electrons residing in the cluster atmosphere - the Sunyaev-Zel'dovich effect (SZE; see Sunyaev \& Zel'dovich 1980; Birkinshaw 1999; Colafrancesco 2007, for reviews). This effect provides a CMB temperature change

$\frac{\Delta T}{T_{0}}=\frac{\sigma_{\mathrm{T}}}{m_{\mathrm{e}} c^{2}} \int_{\ell} \mathrm{d} \ell n_{\mathrm{e}} k_{\mathrm{B}} T_{\mathrm{e}} \cdot g(x)$

that depends on the cluster temperature $T_{\mathrm{e}}$ directly from its amplitude $\propto y=\frac{\sigma_{\mathrm{T}}}{m_{\mathrm{e}} c^{2}} \int_{\ell} \mathrm{d} \ell n_{\mathrm{e}} k_{\mathrm{B}} T_{\mathrm{e}}$ (where $\sigma_{\mathrm{T}}$ is the Thomson crosssection, $m_{\mathrm{e}}$ is the electron mass, $n_{\mathrm{e}}$ is the electron density, $T_{\mathrm{e}}$ is the cluster temperature, the integral is performed along the line of sight $\ell$ ), and from its spectrum $\propto g(x)$, where $x \equiv h v / k_{\mathrm{B}} T_{0}$ is the frequency normalized to the CMB energy, $h$ is the Planck constant, $k_{\mathrm{B}}$ is the Boltzmann constant, and $T_{0}$ is the presentday CMB temperature. The temperature dependence is explicitly included in the function $g(x)$ in terms of the relativistic effects of the Compton scattering that are more prominent in the high frequency region of the SZE spectrum at $v \gtrsim 300 \mathrm{GHz}$ (see Birkinshaw 1999; Colafrancesco et al. 2003; Colafrancesco 2007 , for a review), where it is therefore possible to measure directly the ICM temperature. This is evident looking at the different temperature dependences of the three spectral characteristics of the thermal SZE signal:

i) the minimum in its intensity located at the frequency

$$
\begin{aligned}
& x_{\text {th,min }} \approx 2.265\left(1-0.0927 \theta_{\mathrm{e}}+2.38 \theta_{\mathrm{e}}^{2}\right) \\
&+\tau\left(-0.00674+0.466 \theta_{\mathrm{e}}\right),
\end{aligned}
$$

where $\theta_{\mathrm{e}} \equiv k_{\mathrm{B}} T_{\mathrm{e}} / m_{\mathrm{e}} c^{2}$, whose value depends weakly on the electron spectrum (i.e. on $T_{\mathrm{e}}$ and $n_{\mathrm{e}}$ ) and equals $\sim 2.26$;

ii) the crossover frequency, $x_{0}$, whose value depends on the electron pressure/energy density and optical depth

$x_{\mathrm{th}, 0} \approx a\left(T_{\mathrm{e}}\right)+\tau b\left(T_{\mathrm{e}}\right)$,

where $a\left(T_{\mathrm{e}}\right)=3.830\left(1+1.162 \theta_{\mathrm{e}}-0.8144 \theta_{\mathrm{e}}^{2}\right)$ and $b\left(T_{\mathrm{e}}\right)=$ $3.021 \theta_{\mathrm{e}}-8.672 \theta_{\mathrm{e}}^{2}$, and is found at a frequency $>3.83$ for increasing values of $T_{\mathrm{e}}$ (the value $x_{\mathrm{th}, 0}=3.83$ is found in the non-relativistic limit, or in the limit $T_{\mathrm{e}} \rightarrow 0$ );

iii) the maximum of its intensity located at the frequency

$$
\begin{aligned}
& x_{\mathrm{th}, \max } \approx 6.511\left(1+2.41 \theta_{\mathrm{e}}-4.96 \theta_{\mathrm{e}}^{2}\right) \\
&+\tau\left(0.0161+8.16 \theta_{\mathrm{e}}-35.9 \theta_{\mathrm{e}}^{2}\right),
\end{aligned}
$$

which depends sensitively on the electron population and its energy (momentum) spectrum (see Dolgov et al. 2001; Colafrancesco et al. 2003, 2009, for the case of electrons with a thermal spectrum; see also Colafrancesco et al. 2003; and Colafrancesco 2004, 2005, 2007, 2008b, for the case of electrons with different spectra).

The SZE can be used, therefore, as an alternative probe of the ICM temperature provided that detailed spectral measurements extending to high frequencies can be obtained (see Colafrancesco 2007, for a review; see Colafrancesco et al. 2009).

The linear dependence of the SZE temperature change $\Delta T$ on the electronic density $n_{\mathrm{e}}$ and temperature $T_{\mathrm{e}}$ (see Eq. (1)) makes it possible - in addition - to enhance the sensitivity of the SZE measurements in the outer regions of the cluster while maintaining adequate sensitivity in the inner parts. Therefore, SZE observations could provide a more uniform and spatially extended coverage of the temperature profile in galaxy clusters than X-ray observations.

Almost nothing is known about cluster temperature profiles obtained directly from SZE observations. Present-day and planned SZE observations from both ground-based experiments (multi-band bolometers and interferometers) or upcoming space-borne experiments (e.g., OLIMPO and Planck) require complementary X-ray observations to place tight constraints on the cluster temperature, even though a somewhat more moderate precision on cluster temperature seems to be sufficient to achieve a reasonable statistical knowledge about the CDM power spectrum, in particular $\sigma_{8}$ (Juin et al. 2007).

In this context, we emphasize that the use of X-ray observed quantities in the SZE data analysis is, however, a delicate procedure, because the SZE-derived temperature is weighted by the Compton parameter $y$. For instance, it is known that the derived peculiar velocity is systematically shifted by $\sim 10-20 \%$ for this reason (see e.g., Hansen 2004).

While observations of the SZE are becoming increasingly accurate (e.g., De Petris et al. 2002; Battistelli et al. 2003; Halverson et al. 2009; Staniszewski et al. 2009), most analyses of SZ data are still based on the simplifying assumption of isothermality, or a single temperature plasma. Several studies have also considered the ability of future SZ observations to extract the cluster physical parameters (see, e.g., Knox et al. 2004; 
Aghanim et al. 2005), but always based on the assumption of isothermality.

A desirable solution with the SZE observations is to achieve simultaneous good angular resolution and high sensitivity to deproject the SZE signal properly throughout the entire cluster atmosphere.

We aim to develop an operational procedure to extract the fundamental parameters of the electron populations that produce the SZE, in both thermal and non-thermal plasmas. By applying this procedure, we identify the main instrumental characteristics required to achieve the scientific objectives of the SZE study that we propose to perform.

We discuss a strategy that is more effective in obtaining spatially-resolved temperature measurements based only on SZE observations. This strategy relies on the following data being available: i) spatially resolved spectral measurements of the cluster temperature; ii) measurements of the temperature profile over a wide spatial range to differentiate the cool core region from the outer cluster atmosphere (or regions of different temperature in the overall atmosphere); iii) detailed and independent temperature measurements of wide frequency band coverage and sufficient spectral resolution.

With the same strategy, we also determine whether we can extract the basic parameters of a non-thermal plasma producing a non-thermal SZE that might add to or differ from the thermal SZE produced in a galaxy cluster.

The outline of the paper is as follows. We describe the general theory of the SZE in Sect. 2, and we generalize this theory to the case of clusters with non-uniform T-profiles, such as cool core clusters, in Sect. 3. We describe a fitting procedure for spatially resolved spectroscopic SZE observations in Sect. 4, where we first discuss the case of the thermal SZE in the reference case of the A2199 cluster. The analysis of the non-thermal SZE and the combination of thermal and non-thermal SZE are also discussed, for completeness, in Sect. 5. The non-thermal SZE is important both for the intrinsic study of non-thermal and/or relativistic plasmas in clusters and in determining their role as biases in the study of the thermal plasma (i.e. the ICM). We present in Sect. 6 the application of our study for two specific galaxy clusters: Perseus, which has a cool core, and Ophiuchus, which is approximately isothermal. We discuss our results and summarize our conclusions in the final Sect. 7.

Throughout the paper, we use a flat, vacuum-dominated cosmological model with $\Omega_{\mathrm{m}}=0.3, \Omega_{\Lambda}=0.7$, and $H_{0}=$ $70 \mathrm{~km} \mathrm{~s}^{-1} \mathrm{Mpc}^{-1}$.

\section{The SZE: theory}

To derive a SZE expression that is applicable to a galaxy cluster with spatially varying density and temperature, we need to recall the general SZE formulae.

We use the formalism presented by Wright (1979), and developed subsequently by Birkinshaw (1999), Enßlin \& Kaiser (2000), and Colafrancesco et al. (2003).

We note that Bœhm \& Lavalle (2009) proposed a covariant formalism for the SZE, claiming, erroneously in the first version of their study, that the formalism presented in Wright (1979) and developed by other authors was incorrect. We verified (both numerically and analytically) that the two approaches, in the Thomson limit, are fully equivalent and provide the same results (see Appendix A for details). In addition to our verification, Nozawa \& Kohyama (2009) also showed analytically that the two approaches are fully equivalent, pointing out the errors made by Bœhm \& Lavalle (2009).
The analytic expression for the upscattered CMB spectrum is

$I(x)=\int_{-\infty}^{+\infty} I_{0}\left(x \mathrm{e}^{-s}\right) P(s) \mathrm{d} s$,

where

$I_{0}(x)=2 \frac{\left(k_{\mathrm{B}} T_{0}\right)^{3}}{(h c)^{2}} \frac{x^{3}}{\mathrm{e}^{x}-1}$

with $x=h v / k_{\mathrm{B}} T_{0}$ (see e.g. Colafrancesco et al. 2003).

The function $P(s)$ is given by the following expression

$P(s)=\sum_{n=0}^{+\infty} \frac{\mathrm{e}^{-\tau} \tau^{n}}{n !} P_{n}(s)$,

where

$\tau=\sigma_{\mathrm{T}} \int_{\ell} n_{\mathrm{e}} \mathrm{d} \ell$

is the electron optical depth and

$P_{n}(s)=\underbrace{P_{1}(s) \otimes \ldots \otimes P_{1}(s)}_{n \text { times }}$,

where the symbol $\otimes$ indicates the convolution product, and

$P_{1}(s)=\int_{0}^{\infty} f_{\mathrm{e}}(p) P_{s}(s, p) \mathrm{d} p$.

The function $f_{\mathrm{e}}(p)$ is the electron momentum distribution function (with $p=\beta \gamma$ ) and is normalized to ensure that $\int_{0}^{\infty} f_{\mathrm{e}}(p) \mathrm{d} p=$ 1. The function $P_{s}(s, p)$ is known from the basic physics of the Compton scattering and is given by

$P_{s}(s, p) \mathrm{d} s=P\left(e^{s}, p\right) \mathrm{e}^{s} \mathrm{~d} s$,

where

$$
\begin{aligned}
P(t, p)= & -\frac{3|1-t|}{32 p^{6} t}\left[1+\left(10+8 p^{2}+4 p^{4}\right) t+t^{2}\right] \\
& +\frac{3(1+t)}{8 p^{5}}\left[\frac{3+3 p^{2}+p^{4}}{\sqrt{1+p^{2}}}\right. \\
& \left.-\frac{3+2 p^{2}}{2 p}(2 \operatorname{arcsinh}(p)-|\ln (t)|)\right] .
\end{aligned}
$$

Our aim is to invert Eq. (5) and derive the spectral and spatial parameters of the electron distribution from measurements of the SZE only. We derive an analytical solution to this problem, while in Sect. 4 we present a numerical procedure.

The expression for $\Delta I(x)$ given by a first order approximation in $\tau$ is

$\Delta I(x)=\tau\left[J_{1}(x)-I_{0}(x)\right]$,

where

$J_{1}(x)=\int_{-\infty}^{+\infty} I_{0}\left(x \mathrm{e}^{-s}\right) P_{1}(s) \mathrm{d} s$.

Thus, by using Eq. (10), we can write

$\frac{\Delta I(x)}{\tau}+I_{0}(x)=\int_{0}^{\infty} k(x, p) f_{\mathrm{e}}(p) \mathrm{d} p$,

where

$k(x, p)=\int_{-\infty}^{+\infty} I_{0}\left(x \mathrm{e}^{-s}\right) P_{s}(s, p) \mathrm{d} s$. 
By introducing the definitions

$$
\begin{aligned}
\mathcal{H} f_{\mathrm{e}}(x) & \equiv \int_{0}^{\infty} k(x, p) f_{\mathrm{e}}(p) \mathrm{d} p \\
\tilde{I}_{\tau}(x) & \equiv \frac{I(x)}{\tau}+I_{0}(x),
\end{aligned}
$$

the previous Eq. (15) is given by

$\tilde{I}_{\tau}(x)=\mathcal{H} f_{\mathrm{e}}(x)$,

whose formal solution is given by

$f_{\mathrm{e}}(x)=\mathcal{H}^{-1} \tilde{I}_{\tau}(x)$

with

$\mathcal{H}^{-1} \mathcal{H}=\mathcal{I}$,

where $\mathcal{I}$ is the identity operator.

\section{The SZE for a galaxy cluster with a radial temperature profile}

To apply the formalism described in Sect. 2 to a cluster with a spatially varying temperature and density profiles (i.e., a cluster with a cool core or a non-isothermal cluster), we must consider that:

- Eqs. (5)-(9) still hold. In particular, the expression for the optical depth remains the same because it depends only on the gas density profile and not on the cluster temperature;

- the expression for the function $P(s)$, and its series expansion in terms of powers of $\tau$, remains formally unchanged, because it determines the probability that a $\mathrm{CMB}$ photon experiences a (logarithmic) change in the frequency $s \equiv \ln \left(v^{\prime} / v\right)$ after traversing the whole cluster;

- the expression for the function $P_{1}(s)$ (i.e. Eq. (10)) must be changed. The expression given in Eq. (10) assumes that the electron momentum distribution function $f_{\mathrm{e}}(p)$ is the same throughout the cluster atmosphere. This assumption is incorrect for a cluster with a cool core (or a non-isothermal cluster) since its temperature, and hence the electron momentum distribution, changes with the cluster radius.

We must, therefore, calculate the function $P_{1}(s)$ by taking into account that the CMB photon, while traversing the cluster atmosphere, encounters regions of different temperature and density. To calculate the redistribution function $P_{1}(s)$ appropriately, we can calculate - along the line of sight $\ell$ - the local electron momentum distributions weighted by its local density.

By describing the thermal electron density as

$n_{\mathrm{e}}(r)=n_{\mathrm{e} 0} \cdot g_{\mathrm{e}}(r)$,

it is possible to derive the average density-weighted momentum distribution along the line of sight $\ell$

$\bar{f}_{\mathrm{e}}(p) \equiv \frac{\int_{\ell} f_{\mathrm{e}}\left(p ; T_{\mathrm{e}}(r)\right) g_{\mathrm{e}}(r) \mathrm{d} \ell}{\int_{\ell} g_{\mathrm{e}}(r) \mathrm{d} \ell}$,

where

$f_{\mathrm{e}}\left(p ; T_{\mathrm{e}}\right)=\frac{\eta}{K_{2}(\eta)} p^{2} \exp \left(-\eta \sqrt{1+p^{2}}\right)$ and $\eta=\left(m_{\mathrm{e}} c^{2}\right) /\left(k_{\mathrm{B}} T_{\mathrm{e}}\right)$ and $K_{2}(\eta)$ is the modified Bessel function of second kind (see, e.g., Abramowitz \& Stegun 1965), which ensures the correct normalization such that

$\int_{0}^{\infty} f_{\mathrm{e}}\left(p ; T_{\mathrm{e}}\right) \mathrm{d} p=1$.

The function $\bar{f}_{\mathrm{e}}$ also satisfies the normalization given in Eq. (25), as can be verified by the equation

$$
\begin{aligned}
\int_{0}^{\infty} \bar{f}_{\mathrm{e}}(p) \mathrm{d} p & =\frac{\int_{0}^{\infty} \mathrm{d} p \int_{\ell} f_{\mathrm{e}}\left(p ; T_{\mathrm{e}}(r)\right) g_{\mathrm{e}}(r) \mathrm{d} \ell}{\int_{\ell} g_{\mathrm{e}}(r) d \ell} \\
& =\frac{\int_{\ell} g_{\mathrm{e}}(r) \mathrm{d} \ell \int_{0}^{\infty} \mathrm{d} p f_{\mathrm{e}}\left(p ; T_{\mathrm{e}}(r)\right)}{\int_{\ell} g_{\mathrm{e}}(r) \mathrm{d} \ell}=1,
\end{aligned}
$$

where we use Eq. (25).

We can, therefore, rewrite Eq. (10) for a cluster with a generic temperature distribution by using the averaged electron momentum distribution

$P_{1}(s)=\int_{0}^{\infty} \bar{f}_{\mathrm{e}}(p) P_{s}(s, p) \mathrm{d} p$,

where $\bar{f}_{\mathrm{e}}(p)$ is given by Eq. (23). This function allows us to calculate the frequency redistribution probability for multiple scattering and, hence, the analytic expression of the SZE up to the required approximation order by using Eqs. (5)-(9).

\section{Fitting procedure: the thermal SZE}

Equation (20) cannot be easily solved by analytical methods. We therefore describe a numerical procedure that can derive the cluster temperature profile from observations of the SZE of appropriate spatial and spectral resolution. We consider an instrument that is able to provide high quality SZE spectra in the frequency range $\sim 100-500 \mathrm{GHz}$ obtained with a spatial resolution of $\sim$ arcmin or less. We discuss the feasibility of such spatiallyresolved spectral observations in Sect. 7.

The quantitative analysis that we present in the following refers to the specific case of the cluster A2199 that has a cool core.

We simulate the thermal SZE observation of the cluster A2199 with parameters (density and temperature) taken from the available X-ray observations, and extract the best fit values of the ICM temperature and optical depth from the simulated SZE observations. We consider, for convenience, the first-order approximation to the thermal SZE, and we discuss in Sect. 5 the consequences of using this approximation. The temperature profile obtained from SZE measurements taken at various cluster radii is then deprojected based on the constraint (prior) that the density profile derived from SZE observations is consistent with that inferred from X-ray measurements; we use this procedure, which assumes the X-ray density prior, to ensure a fast enough computing time and allow us to explore the cluster in detail. We note, however, that the density constraint is not necessary, in principle, because the cluster density profile can be obtained, self-consistently, from the optical depth profile measured from SZE observations. This fully consistent procedure requires, however, much longer computing times since the number of free parameters to be fitted to the observations is larger.

For the reference case of A2199 that we now discuss, our analysis included the following steps: i) we considered X-ray information on A2199 available from Chandra observations 


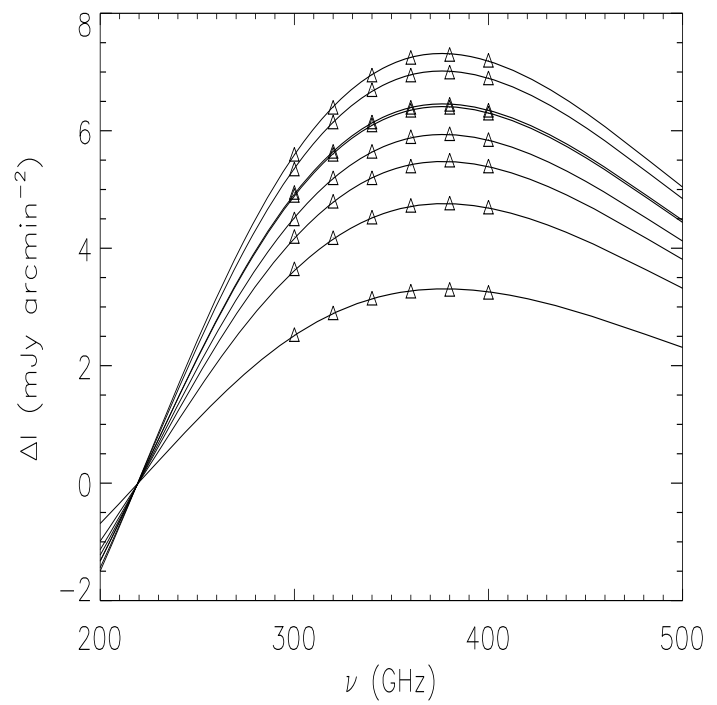

Fig. 1. The SZE spectrum obtained for the cluster A2199 at different projected radii is shown with the frequency sampling derived, for each curve, with a $0.1 \%$ uncertainty. From top to bottom, curves refer to projected radii of $0,5,10,15,20,30,50$, and $100 \mathrm{kpc}$. Note that spectra for 10 and $15 \mathrm{kpc}$ are almost superimposed because beyond this radial distance the IC gas temperature measured by Chandra decreases with increasing radius (Johnstone et al. 2002).

(Johnstone et al. 2002); ii) using the density and temperature profiles derived from X-ray observations, we calculated (using the procedure described in Sect. 3 above) the SZE spectrum at eight different projected radii $r_{\mathrm{p}}$ from the cluster center, i.e., $r_{\mathrm{p}}=0,5,10,15,20,30,50$, and $100 \mathrm{kpc}$, where $1 \mathrm{kpc}$ corresponds to $\approx 1.67$ arcsec at the redshift $z=0.03$ of A2199; iii) for each radial bin, we sampled the calculated SZE spectrum at six different frequencies in the range $300-400 \mathrm{GHz}$, which is the most sensitive part of the SZE spectrum to the cluster temperature, i.e. $v=300,320,340,360,380$ and $400 \mathrm{GHz}$ (see Fig. 1); iv) for each radius, we fitted the six frequency SZE data with a relativistic model of the thermal SZE leaving free two parameters, i.e. $k_{\mathrm{B}} T_{\mathrm{e}}$ and $\tau$, and assuming for each experimental data point an uncertainty of $0.1 \%$ (we also consider below the case of $1 \%$ data uncertainty, for comparison). For each projected radius, this fitting procedure yields a value of the projected temperature and optical depth (see Fig. 2); v) to deproject the fitted temperature profile, we assumed a 3-D theoretical temperature profile of the form

$T_{\mathrm{e}}(r)=T_{\mathrm{int}}+\left(T_{\mathrm{ext}}-T_{\mathrm{int}}\right) \frac{\left(r / r_{\mathrm{c}}\right)^{\mu}}{1+\left(r / r_{\mathrm{c}}\right)^{\mu}}$

(see e.g. Piffaretti et al. 2005). To decrease the number of free parameters in the fit we assumed the ICM density profile $n_{\mathrm{e}}(r)$ obtained from X-ray data. In the fitting procedure, the projected temperature is calculated by weighting the temperature profile by the gas density and integrating along the line of sight to obtain

$T_{\mathrm{e}, \mathrm{p}}\left(r_{\mathrm{p}}\right)=\frac{2 \int_{0}^{R} n_{\mathrm{e}}(r) T_{\mathrm{e}}(r) \frac{r}{\sqrt{r^{2}-r_{p}^{2}}} \mathrm{~d} r}{2 \int_{0}^{R} n_{\mathrm{e}}(r) \frac{r}{\sqrt{r^{2}-r_{p}^{2}}} \mathrm{~d} r}$,

where $R$ is the cluster radius (and it is $R=200 \mathrm{kpc}$ for the case of A2199). Assuming the value of $T_{\text {ext }}$ from $X$-ray observations, the number of free parameters reduces to three. In this way, it is possible to fit the projected temperature obtained from the simulated
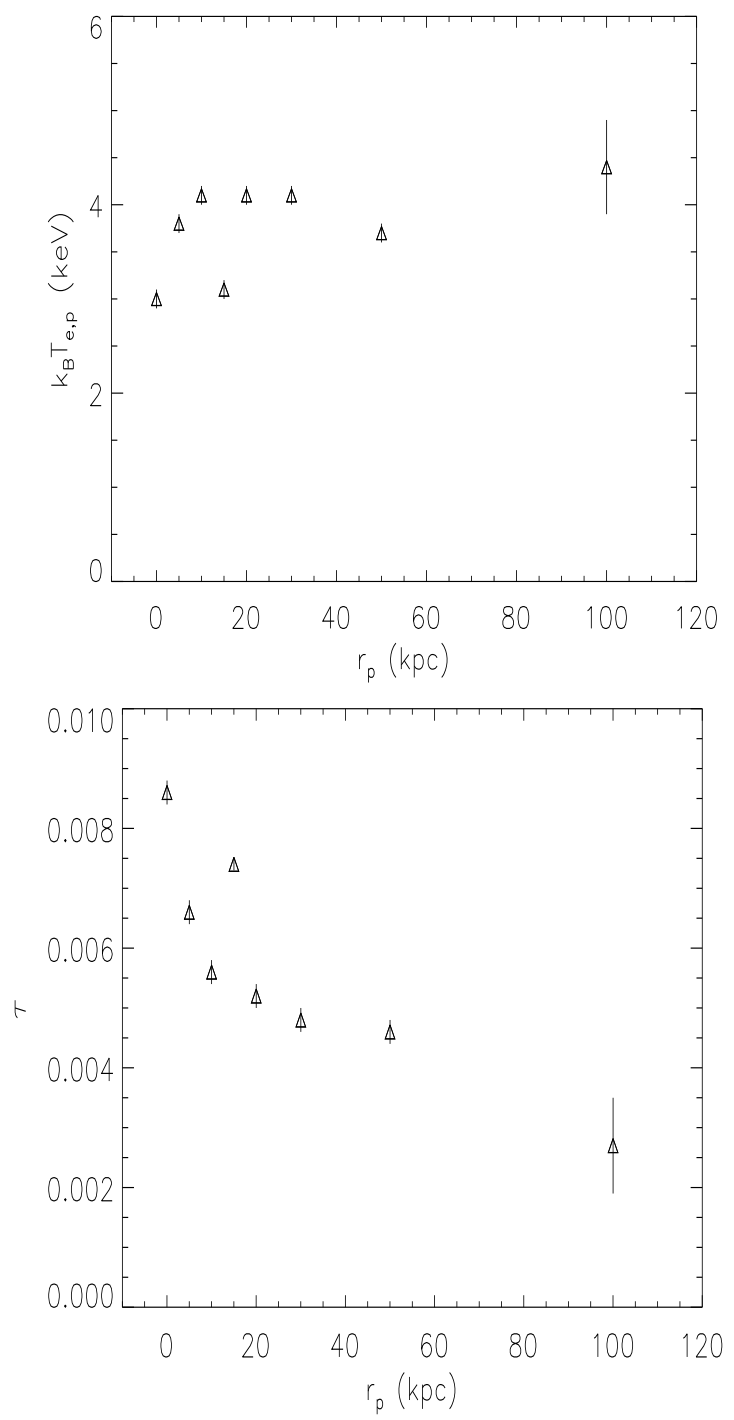

Fig. 2. The radial profile of the projected temperature (top panel) and optical depth (bottom panel) as derived from the fit to the SZE spectra using data sampled in the frequency range $300-400 \mathrm{GHz}$ with uncertainty of $0.1 \%$ (see Fig. 1).

SZE observations, and then derive the temperature profile parameter (in Eq. (28)); assuming $k_{\mathrm{B}} T_{\mathrm{ext}}=4.2 \mathrm{keV}$ for the case of A2199, the best-fit model parameters are $k_{\mathrm{B}} T_{\text {int }}=0.0 \pm 0.5 \mathrm{keV}$, $r_{\mathrm{c}}=4.9 \pm 0.6 \mathrm{kpc}$, and $\mu=1.0 \pm 0.1$. The best-fit temperature profile curve is shown in Fig. 3, where it is compared to the de-projected temperature data derived from X-ray observations.

The previous fitting procedure was repeated assuming that the SZE observations sampled from Fig. 1 have an uncertainty of $1 \%$. The radial profile of the projected temperature obtained in this case is shown in Fig. 4. In this case, the deprojection of the temperature profile using the template given in Eq. (28) does not provide an acceptable fit. We therefore conclude that an uncertainty of order of $1 \%$ in the SZE data is too large to derive a detailed profile of the ICM temperature in clusters like A2199.

The entire fitting procedure was repeated by sampling the SZE observations in the low-frequency range $100-200 \mathrm{GHz}$ $(v=100,120,140,160,180,200 \mathrm{GHz})$ in which the SZE spectrum is, however, much less sensitive to the cluster temperature. In this case, we find that, even assuming uncertainties of $0.1 \%$, it is impossible to reproduce satisfactorily the radial profile of the 


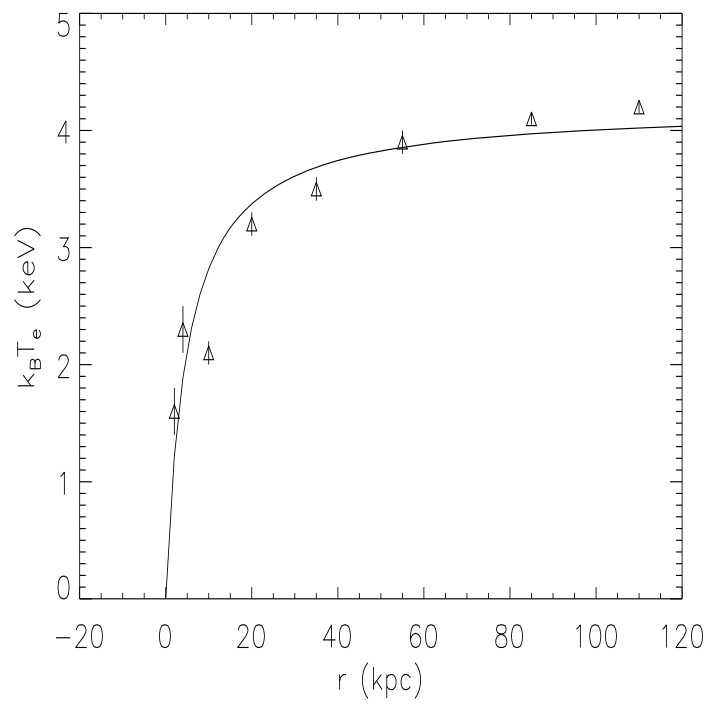

Fig. 3. The deprojected temperature profile curve (solid) derived from SZE spectral data is compared to the deprojected temperature data obtained from X-ray observations of A2199 (Johnstone et al. 2002).

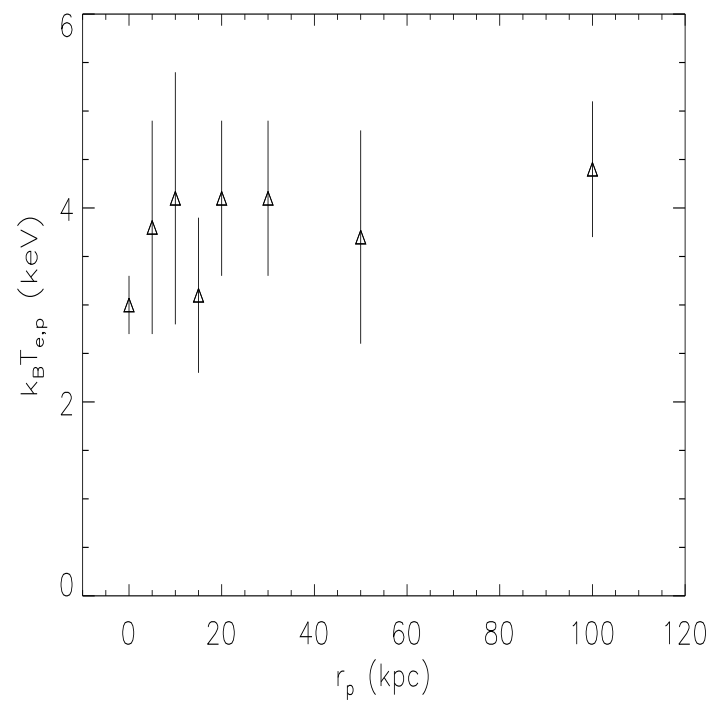

Fig. 4. The projected temperature profile obtained from the fit to SZE spectra using data points sampled in the $300-400 \mathrm{GHz}$ range and assuming an uncertainty of $1 \%$.

projected temperature derived from the fit by using the template in Eq. (28) (see Fig. 5). This is because - as already emphasized - the shape of the SZE spectrum at low frequencies is less sensitive to the cluster temperature than the shape of the highfrequency part of the SZE spectrum. We therefore conclude that, to obtain detailed information about the cluster temperature profile, one must use high-frequency spectral observations of the SZE in the range $300-400 \mathrm{GHz}$. This is crucial when designing the strategy behind SZE observations with the goal of deriving strong constraints on the fundamental parameters of the cluster.

In the frequency range $200-300 \mathrm{GHz}$, which contains the null of the thermal SZE, the kinematic and/or the non-thermal SZE might produce significant additional contributions to the overall SZE. Therefore, in this frequency range, the analysis that we have performed in this section should be performed at a later stage, only after we have estimated the main parameters of the cluster gas, $k_{\mathrm{B}} T_{\mathrm{e}}(r)$ and $n_{\mathrm{e}}(r)$, by using the SZE measurements in the high-frequency range, 300-400 GHz (see Sect. 5.2), which

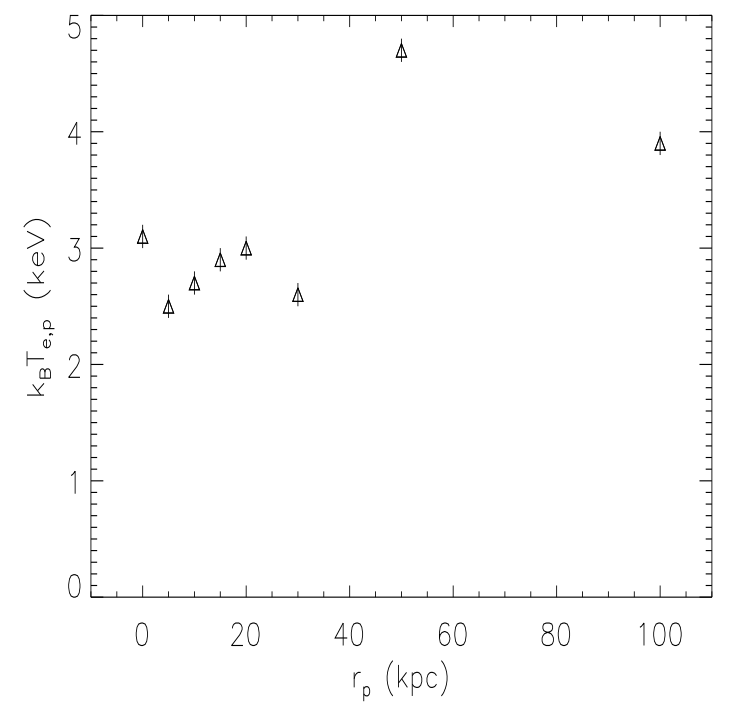

Fig. 5. The projected temperature radial profile obtained from the fit to the SZE spectral data by using data points sampled in $100-200 \mathrm{GHz}$ range and assuming uncertainties of $0.1 \%$.

are much less sensitive to the biases caused by the superposition of the kinematic and non-thermal SZE.

\section{Fitting procedure: the non-thermal SZE}

We simulate the fitting procedure of a non-thermal SZE produced in an extended source such as a cluster radio halo or the lobes of a radio galaxy (see e.g. Colafrancesco 2008) and we consider the effects of the variation in the parameters of the nonthermal electron spectrum on the relative non-thermal SZE.

We first study the spectral shape of the non-thermal SZE as a function of the electron spectral parameters. We use an arbitrary normalization in which the amplitude of the SZE at its minimum is set to be equal to -1 , and we vary the parameters of the electron spectrum. Figure 6 shows the SZE spectrum for a single power-law spectrum

$f_{\mathrm{e}}(p) \propto p^{-s_{1}} ; \quad p \geq p_{1}$

(here $p=\beta \gamma$ is the normalized momentum), where $s_{1}=3.0$, as a function of the minimum momentum $p_{1}$. It is clear that the frequency position of the minimum of the SZE for high values of $p_{1}$ is found around $\sim 160 \mathrm{GHz}$ and does not change for values $p_{1}>5$, while it is considerably lower (of order of $\sim 140 \mathrm{GHz}$ ) only for low values of $p_{1}$, such as $p_{1} \approx 1$. The shape of the spectrum at higher frequencies $(v \geq 300 \mathrm{GHz})$ is, instead, more sensitive to the value of $p_{1}$.

We show in Fig. 7 the case of a double power-law spectrum

$f_{\mathrm{e}}(p) \propto\left\{\begin{array}{cc}p^{-s_{1}} & p_{1} \leq p \leq p_{\text {break }} \\ p_{\text {break }}^{-s_{1}}\left(p / p_{\text {break }}\right)^{-s_{2}} & p>p_{\text {break }}\end{array}\right.$

where $s_{1}=0.1, p_{\text {break }}=100$, and $s_{2}=3.0$. In this case, the position of the minimum and the shape of the spectrum at frequencies $v \leq 400 \mathrm{GHz}$, depends weakly on the value of $p_{1}$, while a stronger dependence from $p_{1}$ is present at higher frequencies (this dependence is weaker than in the single powerlaw case because of the lower power at the low- $p$ end of the electron spectrum).

Figure 8 shows the changes in the SZE spectrum when we consider a single power-law with $p_{1}=1$ as a function of the 
S. Colafrancesco and P. Marchegiani: SZ effect and cluster temperature profiles

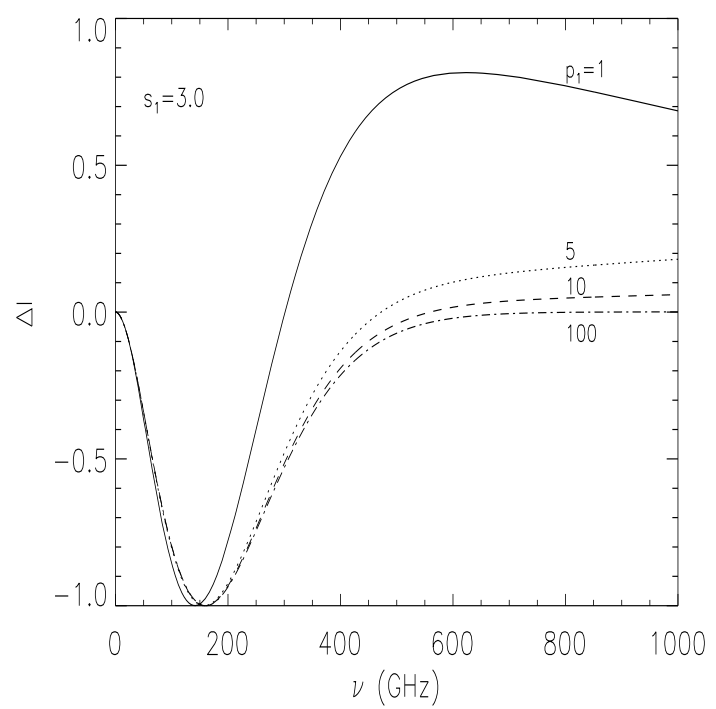

Fig. 6. The non-thermal SZE spectrum, normalized to its minimum amplitude is shown as a function of the minimum momentum $p_{1}$ of the electron spectrum. We assume here a single power-law spectrum with power-law index $s_{1}=3.0$.

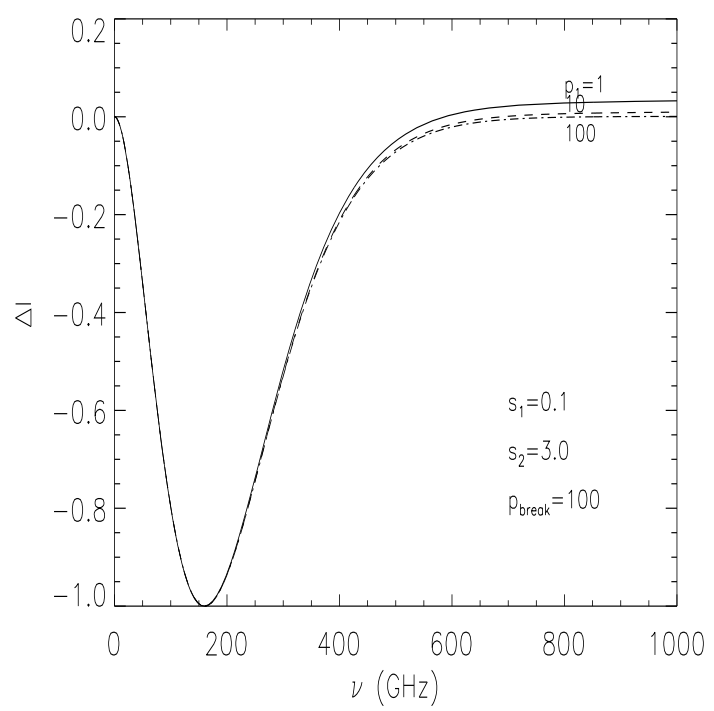

Fig. 7. The non-thermal SZE spectrum, normalized to its minimum amplitude is shown as a function of the minimum momentum $p_{1}$ of the electron spectrum. We assume here a double power-law spectrum with $s_{1}=0.1, s=3.0$, and $p_{\text {break }}=100$. Spectral curves are for $p_{1}=1$ (solid), 10 (dashed), and 100 (dot-dashed).

spectral index $s_{1}$ : the shape of the SZE spectrum is more sensitive to the shape of the electron spectrum at frequency $v \geq$ $400 \mathrm{GHz}$. Figure 9 shows the variation in the SZE spectrum as a function of the spectral index $s_{2}$ for a double power-law spectrum: the dependence on the electron spectrum index is quite weak for the whole frequency range considered.

After illustrating the dependence of the SZE spectrum on the electron spectrum parameters, we discuss the procedures by which we can recover the physical parameters of the nonthermal electron spectrum from the SZE observations. We consider here two cases: i) a pure non-thermal electron population with a power-law spectrum (a situation applicable to radio galaxy lobes, see e.g. Colafrancesco 2008b); ii) a combination of thermal plus non-thermal electron population with a double power-law spectrum (a situation applicable to galaxy

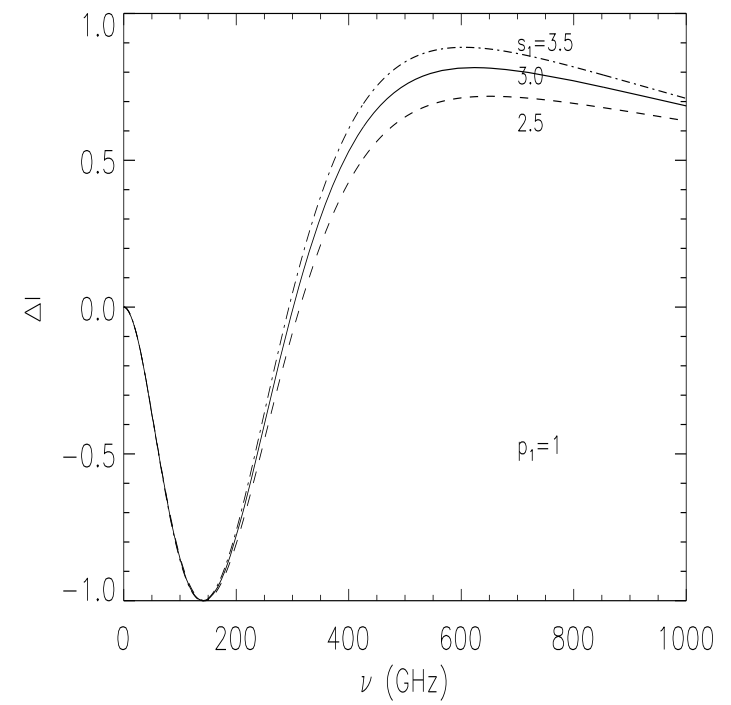

Fig. 8. The non-thermal SZE spectrum, normalized to its minimum amplitude is shown as a function of the spectral index $s_{1}$ of the electron spectrum. We assume here a single power-law spectrum with $p_{1}=1$. Curves are for $s_{1}=3$ (solid), 2.5 (dashed), and 3.5 (dot-dashed).

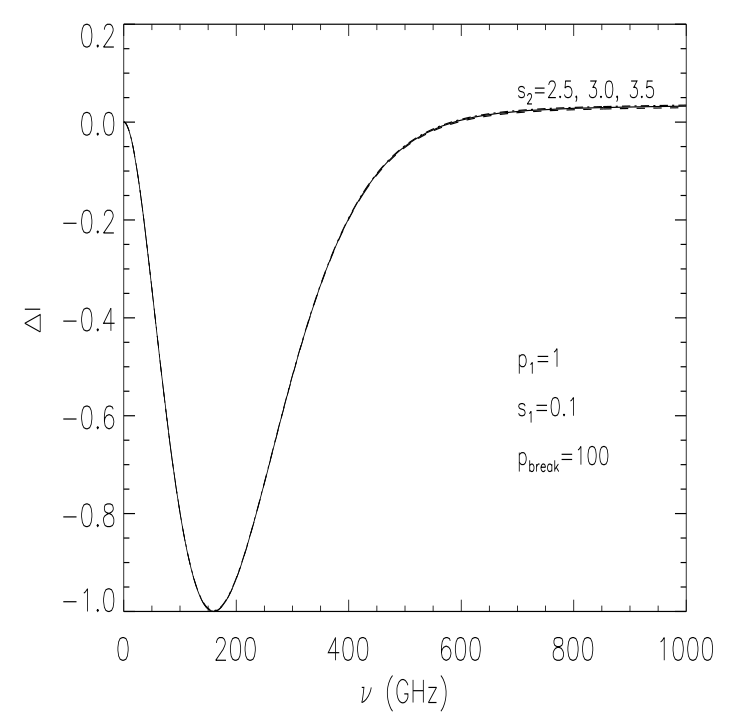

Fig. 9. The non-thermal SZE spectrum, normalized to its minimum amplitude is shown as a function of the spectral index $s_{2}$ of the electron spectrum. We assume here a double power-law spectrum with $p_{1}=1$, $s_{1}=0.1$ and $p_{\text {break }}=100$.

clusters with non-thermal phenomena, see e.g. Colafrancesco et al. 2003).

\subsection{The case of a single power-law spectrum}

We describe how to derive the parameters of an electron population with a single power-law spectrum, assuming that this is the population principally responsible for the SZE production.

Following the procedure previously outlined for the thermal SZE in Sect. 4, we sampled the calculated non-thermal SZE produced at different projected radii and at six different frequencies in the range $300-400 \mathrm{GHz}$, where, as previously noticed, the shape of the SZE is most sensitive to the physical parameters of the electron spectrum. The non-thermal emission region 


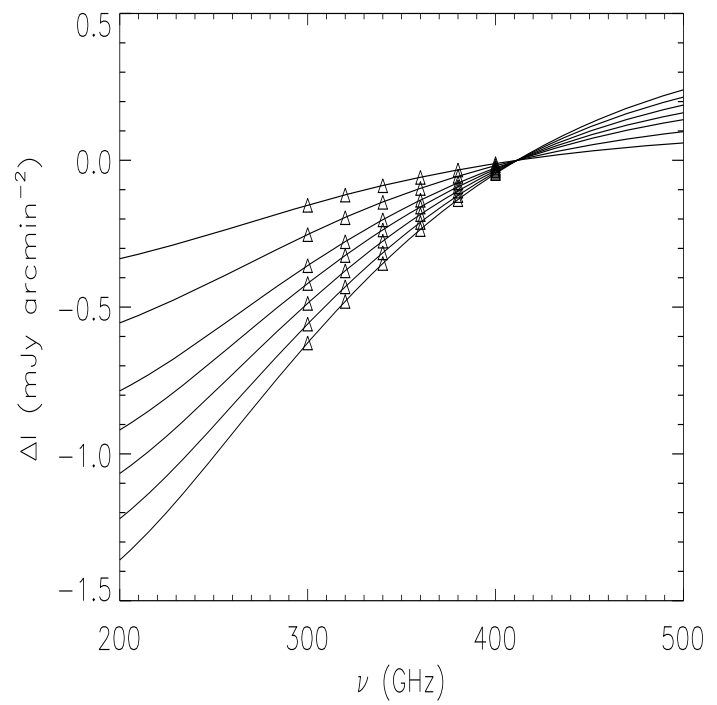

Fig. 10. The non-thermal SZE spectrum evaluated at various projected radii and frequency sampling (see text for details) with data uncertainty of $0.1 \%$. Curves are, from bottom to top, for projected radii of $0,5,10$, $15,20,30,40 \mathrm{kpc}$.

was assumed, for simplicity, to have spherical symmetry, a radius of $50 \mathrm{kpc}$, and an electron spectrum

$N_{\mathrm{e}}(p, r)=k_{0} p^{-s_{1}} \cdot g_{\mathrm{e}}(r) \quad p \geq p_{1}$,

where we assume $s_{1}=3.0, p_{1}=3, k_{0}=10^{-2} \mathrm{~cm}^{-3}$, and a radial distribution of the electron population given by

$g_{\mathrm{e}}(r)=\left[1+\left(\frac{r}{r_{\mathrm{c}}}\right)^{2}\right]^{-q_{\mathrm{e}}}$

with $r_{\mathrm{c}}=10 \mathrm{kpc}$ and $q_{\mathrm{e}}=0.5$. These parameters are typical of giant radio galaxy lobes.

In the fitting procedure, we fixed the spectral index of the electron spectrum $s_{1}$, because it can be derived from radio observations, and we fit the simulated observations with two free parameters: the optical depth $\tau$ and the minimum momentum $p_{1}$. We note that we did not need to assume the parameter $s_{1}$ derived from radio data, because, with adequate frequency coverage, it can be derived from the SZE data alone; this assumption is made for the sole purpose of reducing the numerical computation time.

The SZE intensity at different radii and the relative sampling are shown in Fig. 10. The behaviour of the optical depth at different projected radii, as derived by the fitting procedure, is shown in Fig. 11. Our analysis recovers the input value $p_{1}=3.1 \pm 1.0$ (see Eq. (32)) at all considered projected radii. To deproject the optical depth radial distribution and derive the radial profile of the electron density, it is sufficient to fit the optical depth profile with the density profile given in Eq. (33) and integrate along the line of sight. The deprojection procedure yields $k_{0}=$ $(9.0 \pm 3.5) \times 10^{-3} \mathrm{~cm}^{-3}, r_{\mathrm{c}}=7.2 \pm 6.0 \mathrm{kpc}$, and $q_{\mathrm{e}}=0.43 \pm 0.21$, which are consistent, within the errors, with the input values that we used in the simulations.

We emphasize that the previous analysis of the SZE spectral measurements allowed us to derive directly the density profile and the spectral shape of the electron distribution in the radio galaxy lobes. Therefore, the combination of SZE spectral measurements and the measurement of the low-frequency radio emission allows us to break the degeneracy affecting synchrotron emission between the electron density and the magnetic field and

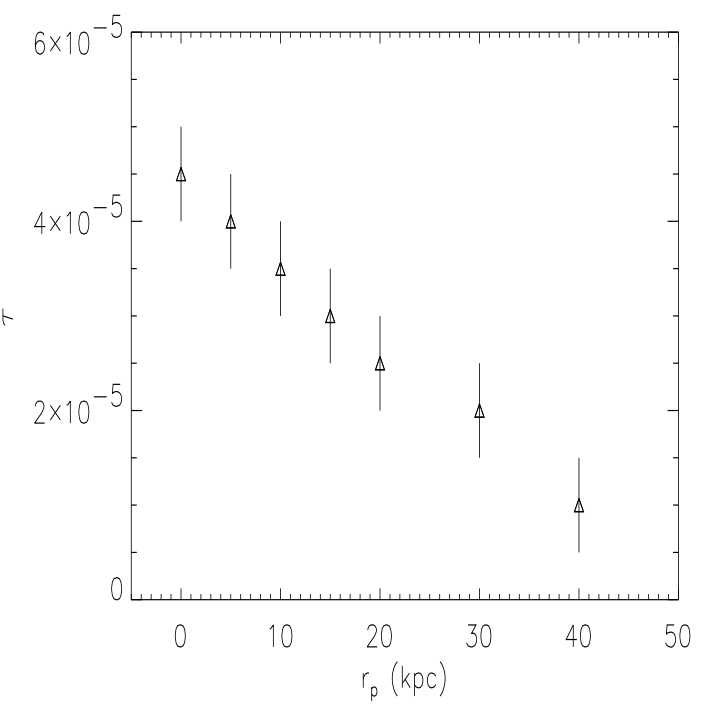

Fig. 11. The radial profile of the optical depth as obtained from the fit to the SZE spectra $b$ using data sampled in the range $300-400 \mathrm{GHz}$ with uncertainty of $0.1 \%$ (see Fig. 10).

eventually yield a precise measure of the magnetic field in radio galaxy lobes (see also Colafrancesco 2008b).

\subsection{The case of thermal plus non-thermal populations}

We consider a combination of a thermal electron distribution that reproduces the A2199 cluster data and an additional nonthermal electron spectrum with a double power-law spectrum, as in Eq. (31), with parameters $s_{1}=0.1, s_{2}=3.0, p_{1}=1$, and $p_{\text {break }}=100$. For the radial (thermal and non-thermal) components, we consider the density profile given in Eq. (33) with parameters $r_{\mathrm{c}}=2.8 \mathrm{kpc}$ and $q_{\mathrm{e}}=0.405$. These parameters accurately reproduce the density profile of A2199 obtained from $\mathrm{X}$-ray observations (see discussion in Sect.4).

A non-thermal electron spectrum similar to a double power law is expected in galaxy clusters because the non-thermal electrons of relatively low energy lose energy by means of Coulomb interactions with the thermal IC gas, and these energy losses flatten the electron spectrum at low $(\$ 150 \mathrm{MeV})$ energies (see, e.g., Colafrancesco \& Marchegiani 2008, 2009, for a discussion). We do not follow a strategy identical to that described in previous sections, in that we do not perform a fit to the sampled points because this procedure would involve too many free parameters (i.e., those of both the thermal and the non-thermal electron populations). We instead focus on the spectral regions where the non-thermal SZE is expected to be more relevant and therefore can be measured. For this purpose, we compare the non-thermal SZE spectrum with both the thermal SZE spectrum, calculated both at first order in $\tau$ and with second order corrections (see Sect. 2 and Colafrancesco et al. 2003, for more details), and the spectrum of a possible SZ kinematic effect. The spectral regions where the non-thermal SZE could be most reliably estimated are i) the frequency range around the minimum of the SZE (at $v \sim 150 \mathrm{GHz}$ ), because all amplitudes are basically proportional to the optical depth of the relative electronic population; and ii) the frequency range around the crossover frequency of the thermal SZE (at $v \sim 220 \mathrm{GHz}$ ), because the thermal SZE is by definition null at this frequency, leaving hence visible the (negative in sign) non-thermal SZE. 


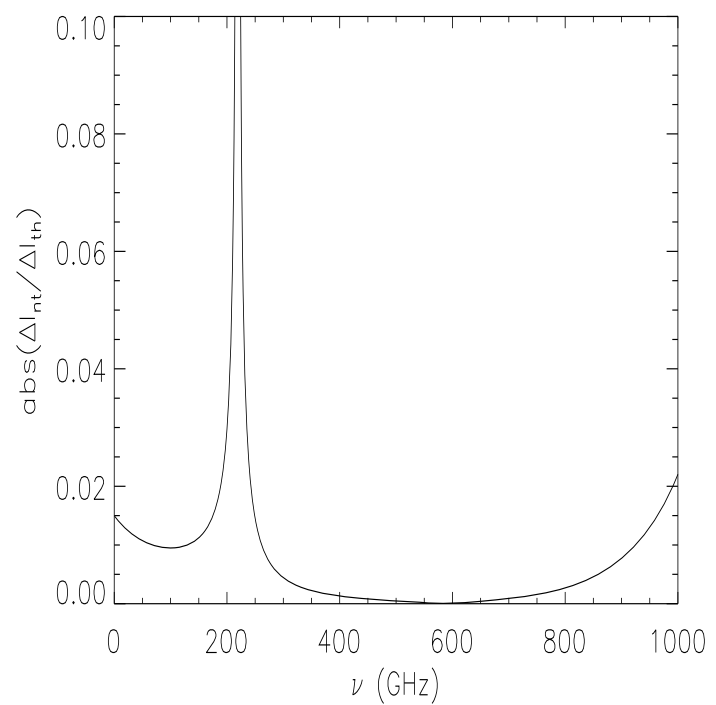

Fig. 12. The frequency dependence of the ratio of the non-thermal SZE (we assume a double power-law spectrum with $s_{1}=0.1, s_{2}=3.0$, $p_{1}=1$, and $\left.p_{\text {break }}=100\right)$ to the thermal SZE at the center of A2199. We assume a pressure balance of $P_{\mathrm{nt}} \sim P_{\mathrm{th}}$ at the cluster center.

We note that an upper limit to the non-thermal electron density can be derived, after fixing their spectral shape, by requiring that the non-thermal pressure does not exceed the thermal one (see discussion in Colafrancesco et al. 2003). Figure 12 shows the ratio of the non-thermal SZE to the thermal SZE in the case $P_{\mathrm{nt}} \sim P_{\mathrm{th}}$ at the cluster center: this case can be considered as an upper limit to the contribution of the non-thermal component to the total SZE. Figure 13 shows the spectrum of the thermal and thermal plus non-thermal SZE in the frequency ranges $100-200 \mathrm{GHz}$ and $300-400 \mathrm{GHz}$. The ratio of the nonthermal to the thermal SZE is large only in the region around $v \sim 220 \mathrm{GHz}$, where the thermal SZE has its crossover and where, consequently, the total SZE is dominated by the nonthermal component. For $v<200 \mathrm{GHz}$, the non-thermal SZE is $\sim 1 \%$ of the thermal one, while for $v>300 \mathrm{GHz}$, the ratio becomes very low, of order of $\sim 0.1-0.5 \%$ or even less. Two conclusions can be derived from these results: 1) in the frequency range $300-400 \mathrm{GHz}$, which is optimal for deriving precise information about the thermal SZE, the non-thermal component does not provide a contribution that jeopardizes the precision of the measurements; 2) at lower frequencies, around $200 \mathrm{GHz}$, the contribution of the non-thermal component is more relevant but, to measure this component, we must differentiate it from the thermal one.

A possible observational means of separating the nonthermal effect from the thermal one consists of measuring the thermal SZE in the frequency range $300-400 \mathrm{GHz}$ and deriving the IC gas physical parameters, as shown in Sect. 4. Then, once the thermal SZE parameters are fixed, it is possible to measure the SZE in the frequency range $100-200 \mathrm{GHz}$ and derive the parameters of the non-thermal population that, in this frequency range, produce a contribution to the total SZE of order of $\sim 1 \%$ (see Figs. 12 and 13): such a SZE amplitude is measurable if measurements have an uncertainty no greater than $\sim 0.1 \%$.

To precisely determine the cluster parameters, we must take into account the contribution of the thermal SZE evaluated at second order in $\tau$, which can be calculated in detail once the main parameters of the IC gas are known (see Colafrancesco et al. 2003, for details). The modifications induced by the second order effect, compared to both the non-thermal SZE and the
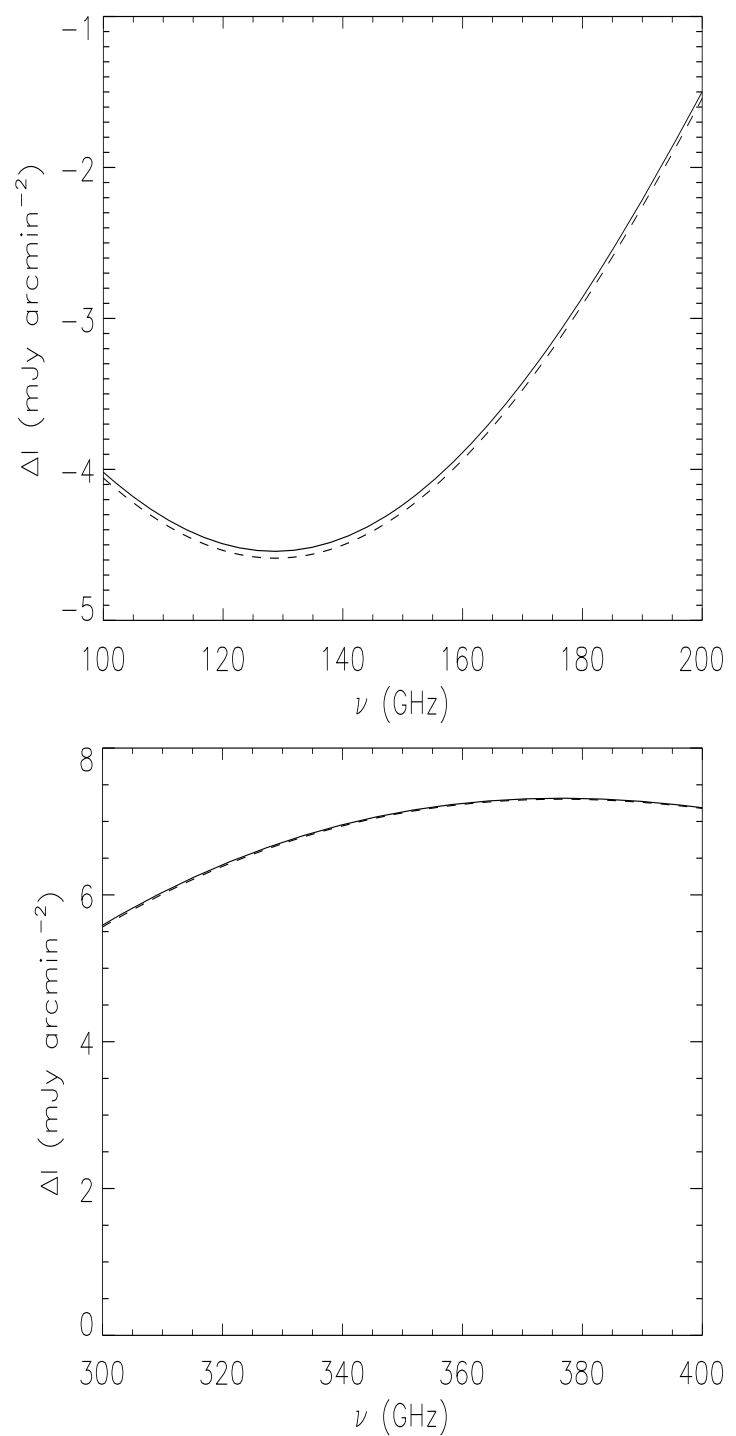

Fig. 13. The thermal (solid curve) and thermal plus non-thermal (dashed curve) SZE evaluated at the center of A2199. The non-thermal electron population has a double power-law spectrum with parameters $s_{1}=0.1$, $s_{2}=3.0, p_{1}=1$, and $p_{\text {break }}=100$ and a pressure $P_{\mathrm{nt}} \sim P_{\text {th }}$ at the cluster center. The spectra are shown in the frequency range $100-200 \mathrm{GHz}$ (upper panel) and 300-400 GHz (lower panel).

first-order thermal SZE, are shown in Fig. 14. The frequency range $300-400 \mathrm{GHz}$ is the optimal frequency window for extracting the thermal SZE parameters, and its second-order correction to the thermal SZE is less than $30 \%$ of the non-thermal SZE and less than $0.1 \%$ of the first-order thermal SZE. Hence, we can conclude that the total SZE can be adequately approximated, in this frequency range, only by the first-order thermal SZE. In the frequency range $100-200 \mathrm{GHz}$, where the nonthermal effect can be measured, the second order correction to the thermal effect is $\sim 5 \%$ of the non-thermal effect, so that the second order correction can also be neglected for this purpose.

We also consider now the possible contribution of a kinematic SZE, whose amplitude is maximal at frequencies close to the crossover frequency of the thermal SZE, i.e. at $v \sim 220 \mathrm{GHz}$. Figure 15 shows the ratio of the kinematical to the non-thermal SZE (upper panel) and the ratio of the kinematical to the thermal SZE (lower panel) for a receding cluster with peculiar velocity of $1000 \mathrm{~km} \mathrm{~s}^{-1}$. In the frequency range $300-400 \mathrm{GHz}$, the result is that the kinematical contribution is a small fraction 

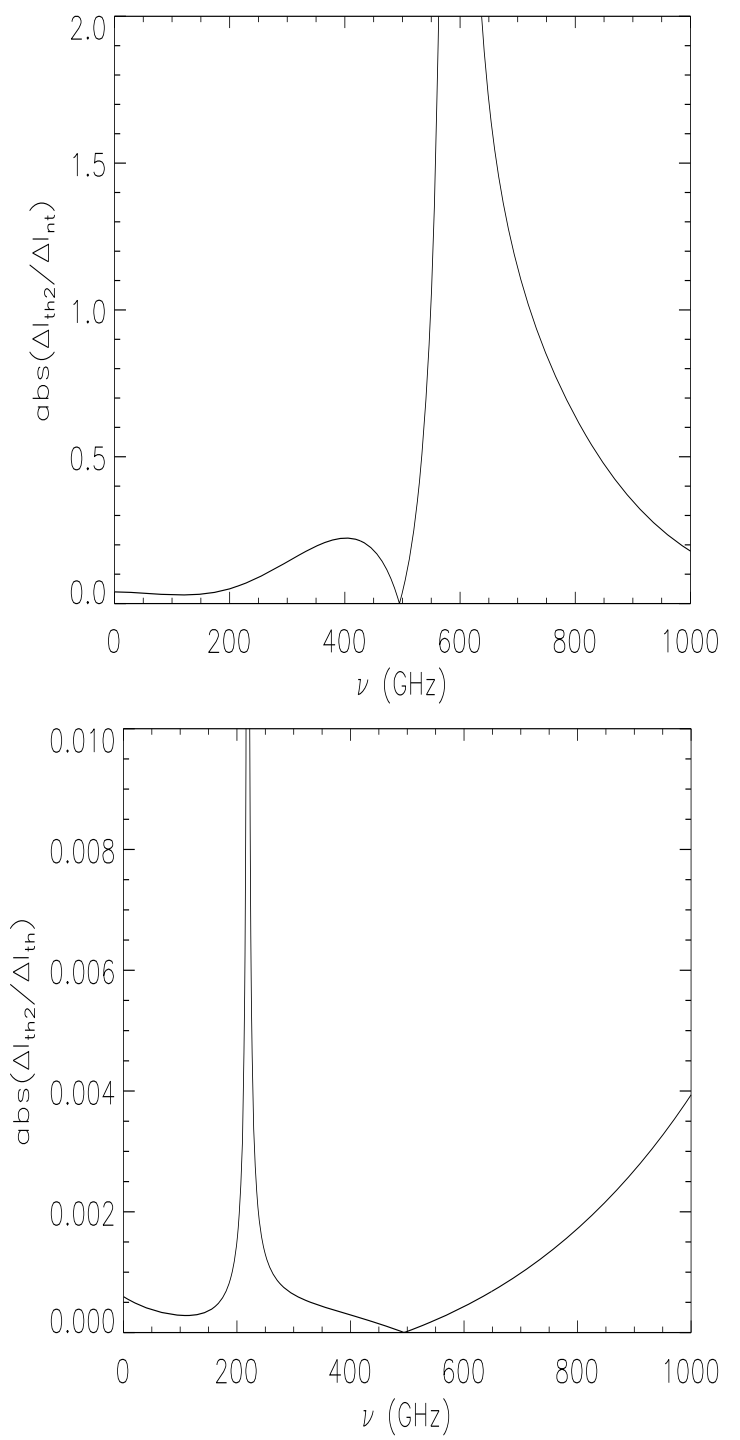

Fig. 14. Upper panel: the frequency dependence of the absolute value of the ratio of the second order correction to the thermal SZE at the center of A2199 to the non-thermal SZE of an electron population that has a double power-law spectrum with parameters $s_{1}=0.1, s_{2}=3.0, p_{1}=1$, and $p_{\text {break }}=100$ and pressure $P_{\mathrm{nt}} \sim P_{\text {th }}$. Lower panel: the frequency dependence of the ratio of the second order correction to the first order description of the thermal SZE.

( $\sim 20 \%$ ) of the non-thermal SZE, and less than $0.1 \%$ of the thermal one. Therefore, the kinematical SZE can also be neglected in this frequency range. Figure 15 also shows that in the range $100-200 \mathrm{GHz}$ the kinematical SZE is less than $10 \%$ of the nonthermal SZE. However, if the non-thermal electron population were to have a pressure $P_{\mathrm{nt}} \sim 0.1 P_{\mathrm{th}}$, then the non-thermal and kinematical SZE would be comparable (based on the assumption of $V_{\mathrm{p}}=1000 \mathrm{~km} \mathrm{~s}^{-1}$ ); in this case, however, these signals would be indistinguishable from the thermal one (for reference see Figs. 12 and 13 upper panel, in which we show the case $\left.P_{\mathrm{nt}} \sim P_{\mathrm{th}}\right)$.

Our results can be summarized as follows:

i) in the frequency range $300-400 \mathrm{GHz}$, the first order thermal SZE is a good approximation of the total SZE (all the corrections are $\leq 0.1 \%$ ).

ii) in the frequency range $100-200 \mathrm{GHz}$, if the non-thermal contribution is strong, it dominates over the kinematic one.
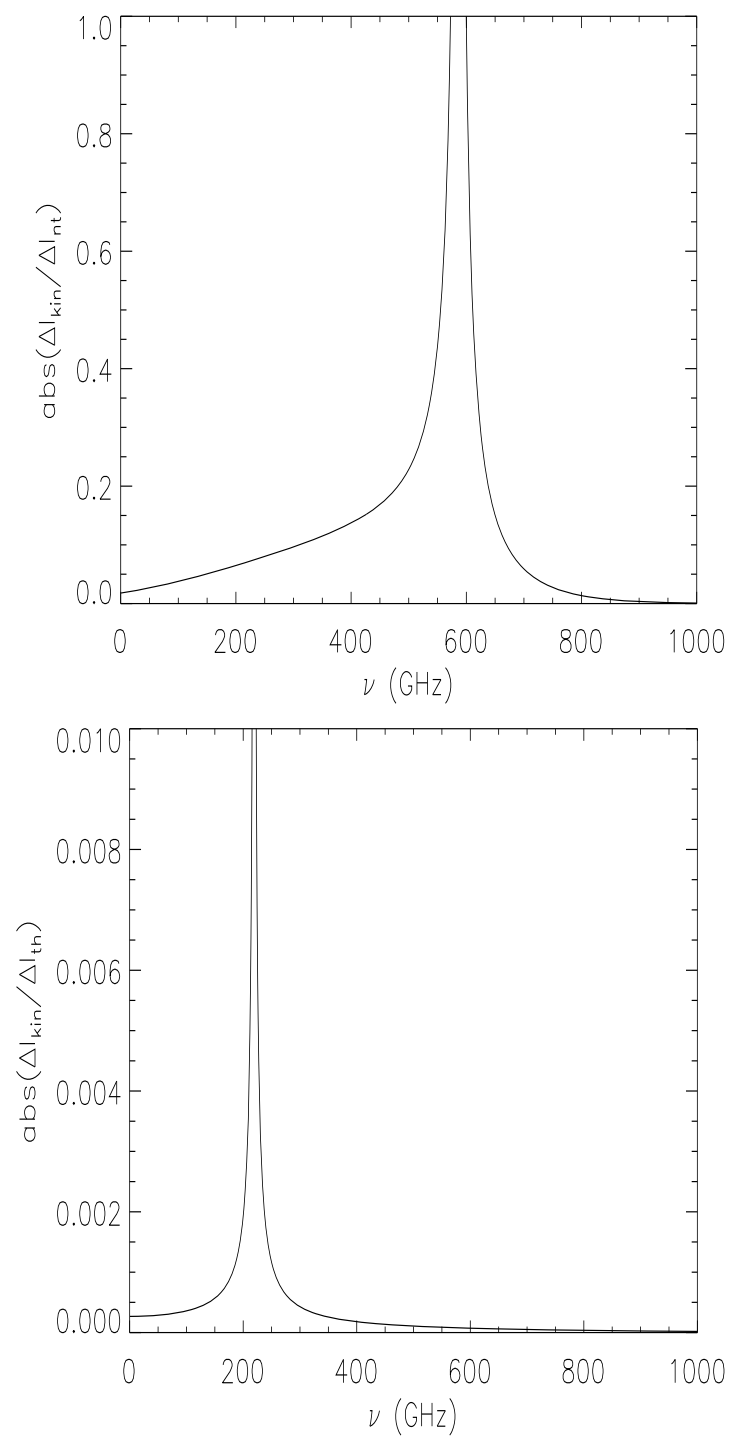

Fig. 15. Upper panel: the frequency dependence of the absolute value of the ratio of the kinematic SZE for a value of the peculiar velocity of $v_{\mathrm{p}}=$ $1000 \mathrm{~km} \mathrm{~s}^{-1}$ to the non-thermal SZE for an electron population with the same spectrum as in Fig. 14. Lower panel: the frequency dependence of the absolute value of the ratio of the kinematic SZE to the thermal SZE evaluated to first order.

If, instead, the two SZE contributions are comparable, then they cannot be easily differentiated from the thermal one. Hence, if deviations from expected thermal SZE are measured in this frequency range, they should be of non-thermal origin;

iii) at $v \sim 220 \mathrm{GHz}$ (i.e. the crossover frequency of thermal SZE) alone, the kinematic SZE, the non-thermal SZE and the second order thermal correction are important with respect to the first order thermal effect. To differentiate between the three different contributions, it is important to place constraints on the thermal and the non-thermal parameters by performing precise observations in frequency ranges 300-400 and 100-200 GHz, respectively.

\section{Applications to specific clusters: Perseus and Ophiuchus}

We present our studies of two nearby and extended clusters: Perseus, which has a cool core, and Ophiuchus, which is 


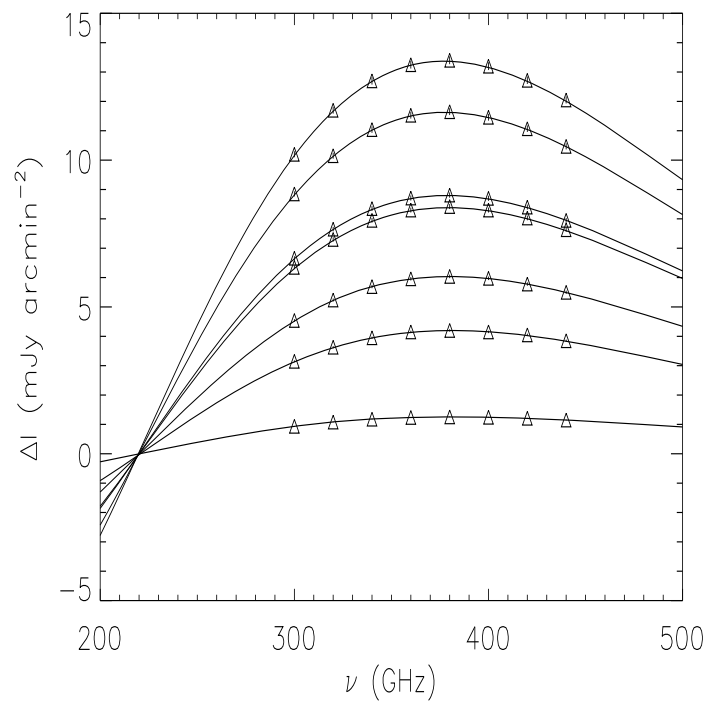

Fig. 16. The thermal SZE spectra of the Perseus cluster evaluated at different projected radii of $0,20,50,75,100,150$, and $200 \mathrm{kpc}$ (from top to bottom) are shown in the frequency range $200-500 \mathrm{GHz}$. For each curve, the SZE sampling in the frequency range $300-450 \mathrm{GHz}$ has an uncertainty of $1 \%$.

approximately isothermal. As discussed previously, the optimal strategy for extracting the physical parameters of the cluster is to consider the frequency range $300-400 \mathrm{GHz}$; in this range, we can safely consider only the first order thermal SZE in our analysis, because higher-order corrections are negligible.

\subsection{The Perseus cluster}

The Perseus cluster is at a redshift $z=0.0179$ or a distance of $77.7 \mathrm{Mpc}$, at which 1 arcmin corresponds to $21.8 \mathrm{kpc}$. Therefore, a study of the spatially resolved SZE allows us to achieve good spatial sampling of the temperature profile, superior to that of the cluster A2199 previously discussed. In the following, we repeat our analysis for this cluster.

Churazov et al. (2003) derived an analytical approximation to the radial profile of the IC gas density and temperature that reproduces the cluster data for $r>10 \mathrm{kpc}$, while at shorter distances the central dominating galaxy NGC 1275 causes the IC gas radial density profile to be more uncertain. For the sake of illustration, we use the analytical fitting formulae given by Churazov et al. (2003) and estrapolate them to the cluster center, ignoring, in this way, the presence of the central galaxy.

We hence simulated to first order the thermal SZE expected for Perseus at seven projected radii from the cluster center: 0 , $20,50,75,100,150$, and $200 \mathrm{kpc}$ (corresponding to angular sizes of $0,0.9,2.3,3.4,4.7,6.9$, and 9.2 arcmin). We then sampled each one of the SZE spectra at these radii for eight different frequencies in the 300-450 GHz range: 300, 320, 340, 360, 380, 400, 420, and $440 \mathrm{GHz}$ (see Fig. 16). We extended here the frequency coverage of the SZE spectra to improve our sampling of the spectral region of the SZE where relativistic effects are relevant. For each projected radius we fitted, using the gas temperature and optical depth as free parameters, the SZE simulated signal by using the expression of the first-order thermal SZE and assuming an error of $1 \%$ in the experimental data. The results of the fit to the projected temperature are shown in Fig. 17. The deprojection procedure of the best-fit radial temperature profile is performed by using Eq. (28), defining $k_{\mathrm{B}} T_{\text {ext }}=6.8 \mathrm{keV}$ (the best-fit projected temperature at $200 \mathrm{kpc}$ ),

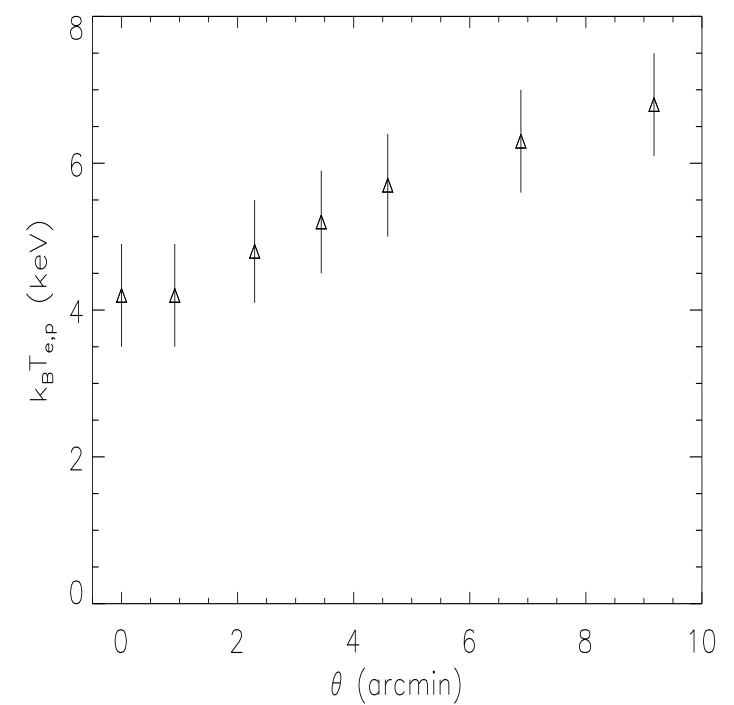

Fig. 17. The radial profile of the projected temperature, derived from the fit to the SZE spectra in Perseus in the frequency range $300-440 \mathrm{GHz}$ with uncertainties of $1 \%$ (see Fig. 16), is shown as a function of the projected radius (in arcmin).

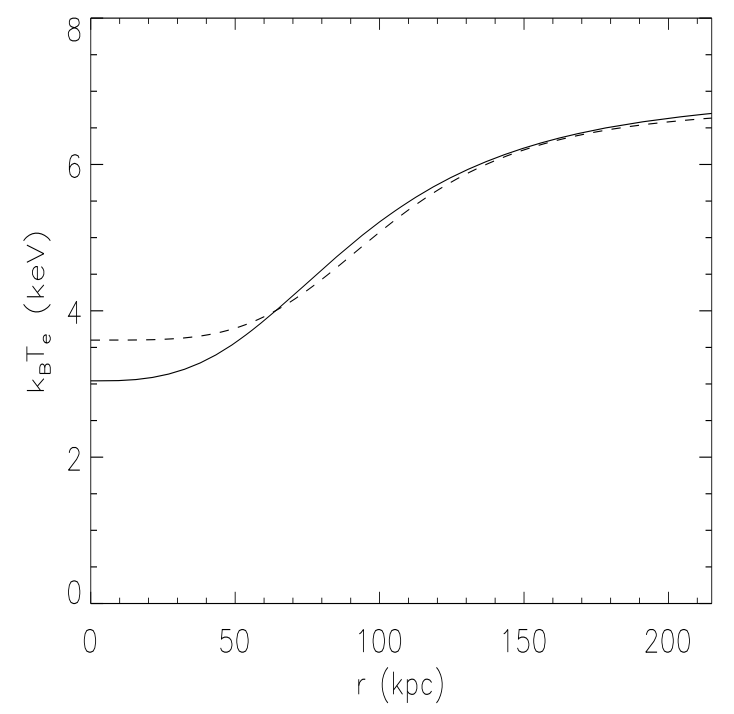

Fig. 18. The deprojected temperature radial profile in Perseus derived from the fit to the X-ray data by Chrurazov et al. (2003) (solid curve) is compared to the fit to the SZE simulated data (dashed curve) shown in Fig. 17. For the SZE temperature profile, we use the radial profile given by Eq. (28), with $k_{\mathrm{B}} T_{\mathrm{ext}}=6.8 \mathrm{keV}, k_{\mathrm{B}} T_{\mathrm{int}}=3.6 \mathrm{keV}, r_{\mathrm{c}}=104 \mathrm{kpc}$, and $\mu=4.0$.

and by using as prior constraints the information about the electron radial profile provided by X-ray observations (see Sect. 4). For an experimental uncertainty of $1 \%$, such procedure yields values $k_{\mathrm{B}} T_{\mathrm{int}}=3.6 \pm 1.5 \mathrm{keV}, r_{\mathrm{c}}=104 \pm 45 \mathrm{kpc}, \mu=4.0 \pm 2.4$, where $\chi^{2}=0.1$ for 4 d.o.f. Figure 18 compares the deprojected temperature profile found with our method from SZE observations with the best-fit deprojected temperature profile derived by Churazov et al. (2003) obtained from X-ray data. The two curves are quite similar except for the inner region, where the IC gas is cooler and the sensitivity of the SZE spectrum to low temperatures (because of relativistic corrections) is lower. We also verified that by assuming an uncertainty of $0.1 \%$ in the experimental data, the best-fit values do not change, but the relative errors are reduced considerably (see Fig. 19). 


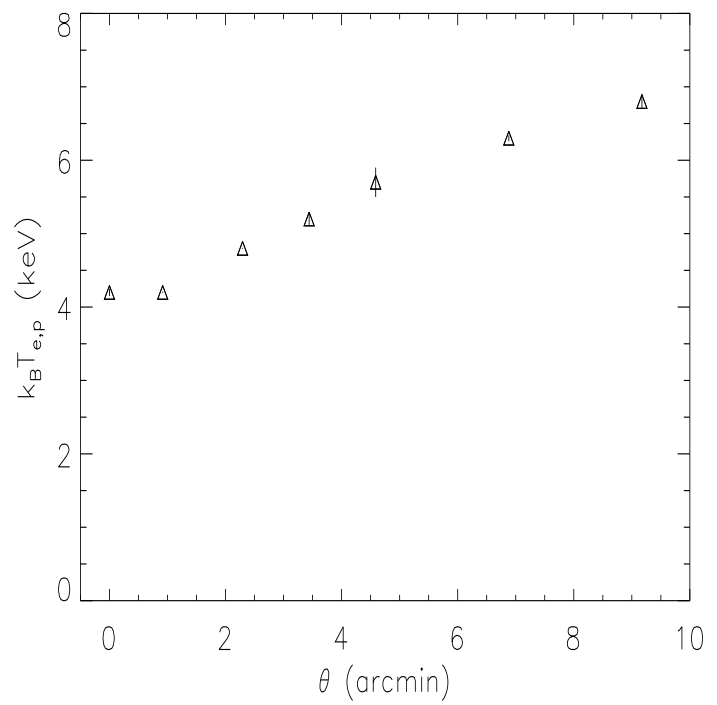

Fig. 19. As Fig. 17 but with uncertainties assumed at the level of $0.1 \%$ of the sampled SZE data points (see Fig. 16).

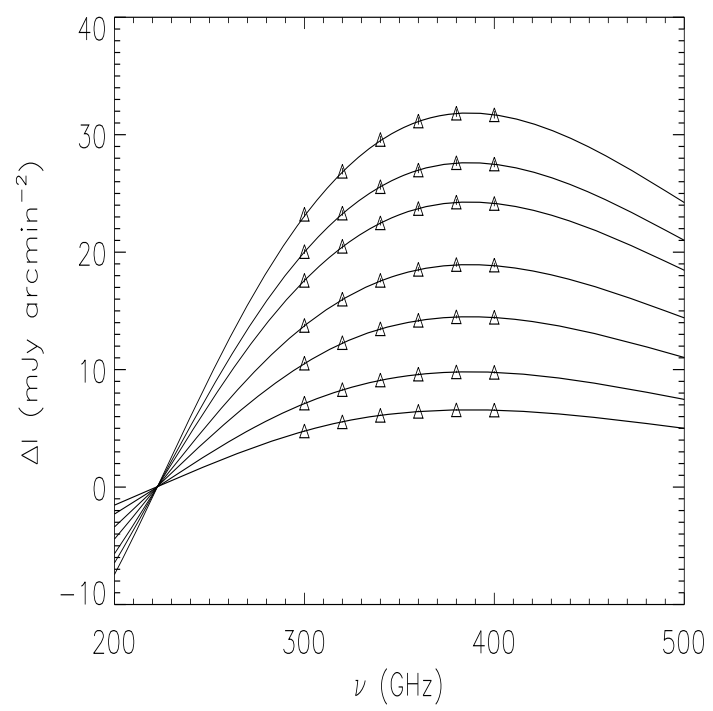

Fig. 20. The thermal SZE spectrum for the Ophiuchus cluster is shown at various projected radii of $0,35,70,120,200,300$ and $500 \mathrm{kpc}$ (from top to bottom). The assumed uncertainty in the SZE data points is $1 \%$.

\subsection{The Ophiuchus cluster}

To first approximation, the Ophiuchus cluster is an isothermal cluster with $k_{\mathrm{B}} T_{\mathrm{e}} \sim 9.9 \mathrm{keV}$ (Watanabe et al. 2001). Since Ophiuchus is quite a hot cluster, relativistic effects in the SZE spectrum are expected to be more prominent, allowing us to obtain a more robust estimate of both the cluster gas temperature and density.

The SZE signal produced in Ophiuchus, calculated as described in Sect. 2, was sampled at six frequencies between 300 and $400 \mathrm{GHz}$ and at seven different radii, 0, 35, 70, 120, 200, 300, and $500 \mathrm{kpc}$ (see Fig. 20), assuming experimental uncertainties of $1 \%$. The minimum radius at which we sampled the radial profiles was chosen by taking into account that at the Ophiuchus distance $(z=0.028), 1$ arcmin corresponds to $\sim 34 \mathrm{kpc}$.

Figure 21 shows the radial profiles of the projected temperature and optical depth of the IC gas obtained from the fit to SZE data. These results clearly indicate that the cluster
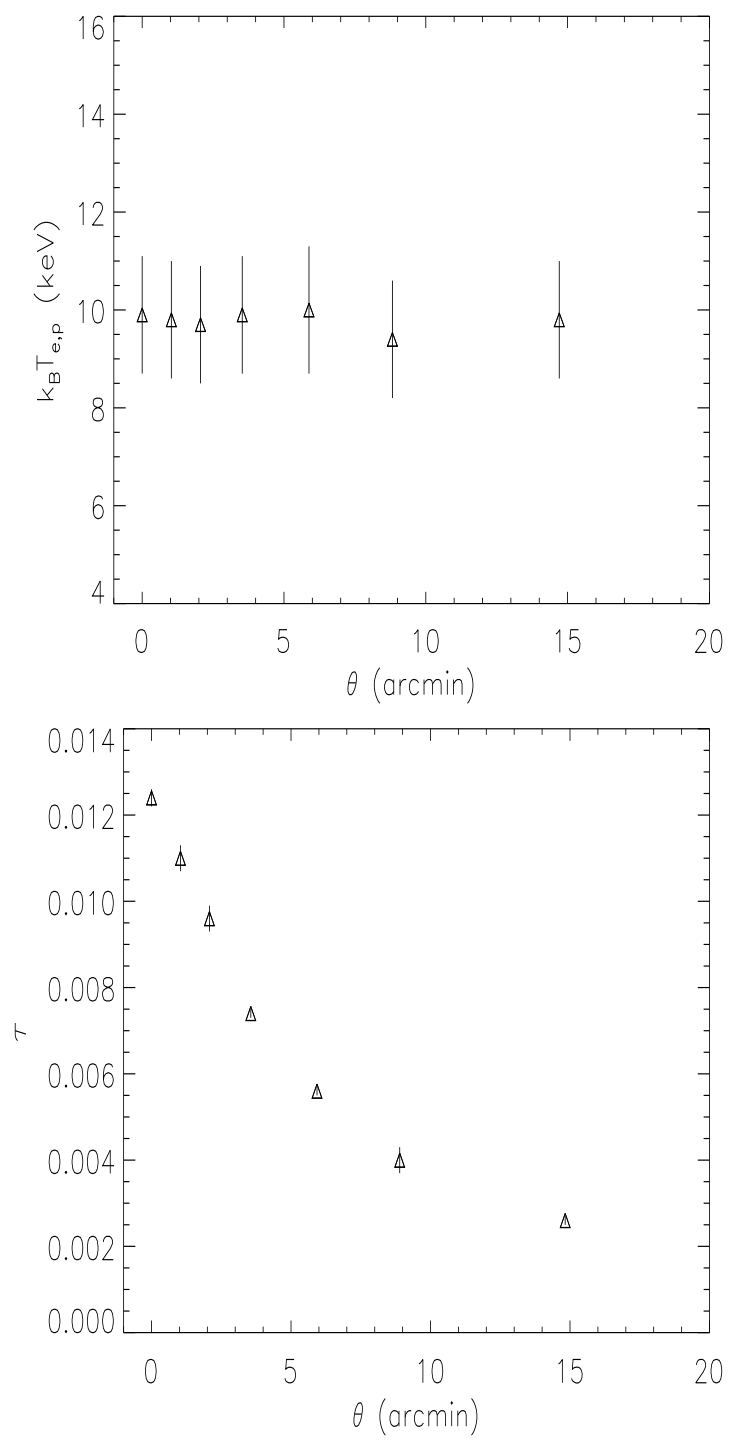

Fig. 21. The radial profile of the projected temperature (upper panel) and of the IC gas density (lower panel) as derived from the fit to the SZE spectra for Ophiuchus shown as a function of the projected radius (in arcmin). We use SZE data points in the frequency range $300-400 \mathrm{GHz}$ with uncertainties of $1 \%$ (see Fig. 20).

temperature is constant with radius, within the errors, while the radial density profile can be derived from the optical depth radial profile by using the model

$n_{\mathrm{e}}(r)=n_{\mathrm{e} 0}\left[1+\left(\frac{r}{r_{\mathrm{c}}}\right)^{2}\right]^{-q_{\mathrm{th}}}$

We therefore note that in a cluster with constant temperature profile, deprojection can be performed using only optical depth results without assuming any prior constraint obtained from X-ray data. Hence, the thermal gas parameters derived from SZE and $\mathrm{X}$-ray data can be directly compared.

The values derived from X-ray measurements are $n_{\mathrm{e} 0}=$ $1.77 \times 10^{-2} \mathrm{~cm}^{-3}, r_{\mathrm{c}}=108 \mathrm{kpc}$, and $q_{\mathrm{th}}=0.96$ (Johnston et al. 1981; Watanabe et al. 2001). The values that we derive from the fit to the simulated SZE signals are $n_{\mathrm{e} 0}=(1.7 \pm 0.2) \times 10^{-2} \mathrm{~cm}^{-3}$, $r_{\mathrm{c}}=118 \pm 2 \mathrm{kpc}$, and $q_{\mathrm{th}}=1.00 \pm 0.07$. The comparison between the best-fit profile to X-ray data and that to the SZE data is shown in Fig. 22. We can see that the density profile derived 


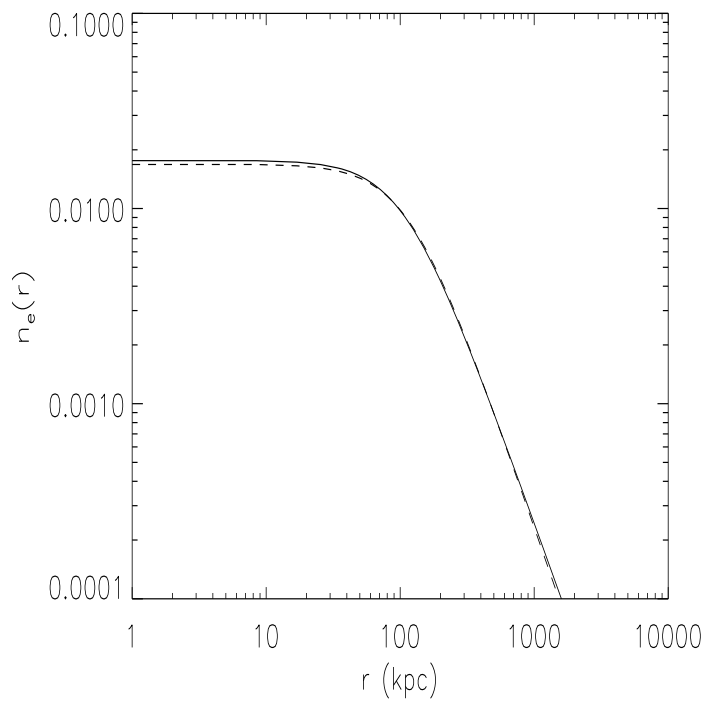

Fig. 22. The IC gas density profile of Ophiuchus derived from X-ray observations (solid curve) compared with that derived from the optical depth radial profile shown in Fig. 21 (dashed line) obtained from SZE simulated observations (see text for details).

from SZE measurements is very similar to that obtained from $\mathrm{X}$-ray observations.

\section{Discussion and conclusions}

We have derived a general formalism to describe the thermal SZE in galaxy clusters with a non-uniform temperature profile, which can be applied to both cool-core clusters and noncool core cluster with isothermal or non-isothermal temperature structures.

To extract the temperature profile information from spatiallyresolved, spectroscopic SZE observations, we have developed an inversion technique by which the electron distribution function can be extracted using a SZE signal with a wide frequency range coverage. We have presented extensive results of the fitting procedure used to extract the cluster gas temperature from a set of simulated spatially resolved spectroscopic SZE observations in different bands of the spectrum, from 100 to $450 \mathrm{GHz}$, and we discuss the optimal experimental and theoretical strategy.

Our analysis of three different cluster prototypes (A2199 with a low-temperature cool core, Perseus with a relatively high-temperature cool core, and Ophiuchus with an isothermal temperature distribution) inferred the required precision of the SZE observations and the optimal frequency bands to determine the cluster gas temperature with an accuracy similar to or better than that obtainable from X-ray observations. The precision level of SZE-derived temperature was also discussed for the outer regions of clusters, which are particularly relevant to accurate mass determination of galaxy clusters. We also investigated how to extract from our method the parameters characterizing the non-thermal SZE spectrum of the relativistic plasma contained in various astrophysical environments, such as the lobes of radio galaxies and the relativistic electrons cospatially distributed with the thermal plasma in galaxy clusters with non-thermal phenomena (e.g., radio halos and/or hard $\mathrm{X}$-ray excesses).

Based on our results, we conclude that the method we present here is the only method, so far, for SZE observations that is able to extract the crucial parameters of the cluster atmospheres (i.e. their temperature, density and additional non-thermal components) using only a single observational technique, i.e. spatially resolved spectroscopic SZE observations. These SZE observations are becoming available with the advent of new, but challenging, experimental techniques.

To achieve a wide spectral range (i.e. the $\sim 100-500 \mathrm{GHz}$ range discussed in our analysis) with imaging capabilities, a Fourier Transform spectrometer would be an optimal experimental solution. Even though FT spectrometers already operate on Herschel(-Spire), specific technical solutions for space-borne projects using Martin-Puplett architecture (Martin \& Puplett 1970) have been presented (see, e.g., DeBernardis et al. 2010). It has been shown in fact that these experimental solutions allow a clean differentiation of the spectra coming from two independent inputs covering two contiguous areas of the telescope focal plane, so that the measured spectrum is the difference in the spectra of the target source and an offset reference field (see, e.g., DeBernardis et al. 2010). For this differential configuration, even small SZE signals can be extracted from a strong mode background generated, e.g., by the CMB itself, and the intrinsic emission from the telescope (either radiatively cooled to $\sim 80 \mathrm{~K}$, or actively cooled to a few $\mathrm{K}$ ), in addition to other signals from the Earth and other astronomical foreground sources.

Fourier Transform spectrometer experiments have the crucial advantage (with respect to dispersion spectrometer experiments) of being imaging instruments, an essential requirement for the analysis we have presented in this paper. With this choice, hence, a large detector array can be arranged in the focal plane of a sub-mm telescope, thus increasing the mapping speed.

To limit the radiative background (and the corresponding fluctuations) of the detectors, and match the detector size to the diffraction-limited angular resolution (which improves with frequency), the total spectral range of interest can be divided into different frequency bands. The frequency ranges of these bands must then be optimized to achieve optimal performance when studying the SZE.

The bands resulting from the SZE study optimization are $B_{1}=100-200 \mathrm{GHz}, B_{2}=200-300 \mathrm{GHz}$, and $B_{3}=$ $300-450 \mathrm{GHz}$. An additional fourth band, $B_{4}=600-800 \mathrm{GHz}$, could be useful in controlling the spectral behaviour of the foreground emission.

The low-to-medium resolution configuration (i.e., a 10 to $20 \mathrm{GHz}$ resolution, but constant over a large frequency band) for the spectrometer envisaged here is completely suitable to study the smooth features of the continuum SZE spectra. We note that the different spectral resolutions (from 20 to $1 \mathrm{GHz}$ ) achievable by Fourier Transform spectrometer enable a wide range of astrophysical sources to be studied.

With a $3 \mathrm{~m}$ diameter dish, the corresponding angular resolution, which is limited by diffraction at the telescope entrance aperture, of the previous three bands used to measure the SZE are approximately $4.5,2.25$, and $1.5 \operatorname{arcmin} F W H M$, respectively. With a $12 \mathrm{~m}$ diameter dish, the angular resolution can be reduced to approximately 1.06, 0.56 and $0.38 \operatorname{arcmin} F W H M$, respectively.

For an instrument field of view (FOV) of $D \times D \operatorname{arcmin}^{2}$ (resulting in two contiguous FOVs in the sky in the differential imaging spectrometer strategy) filled with diffraction limited detectors, the resulting number of detectors for each of the above frequency bands are approximately $(D / 4.5)^{2}\left[(D / 1.06)^{2}\right]$, $(D / 2.25)^{2}\left[(D / 0.56)^{2}\right]$, and $(D / 1.5)^{2}\left[(D / 0.38)^{2}\right]$ for the $3 \mathrm{~m}$ (12 m) diameter dish, respectively.

These optimal spectroscopic capabilities must be coupled to high-sensitivity detectors to fully exploit the large amount of information enclosed in the SZE signal. The photon background depends on the cooling of the whole system: it is dominated by 
the warm telescope $(\sim 80 \mathrm{~K})$ for a passive cooling, but can be strongly reduced by using active cooling that could bring the telescope to a few $\mathrm{K}$. The achievable photon noise limited NEP of each of the detectors can be in the range $\sim 10^{-17} \mathrm{~W} / \sqrt{\mathrm{Hz}}$ to $\sim 10^{-19} \mathrm{~W} / \sqrt{\mathrm{Hz}}$ for a passive or active cooling, respectively, therefore allowing full exploitation of even the smallest SZE signals.

To summarize, the analysis of the SZE signals that we have presented in this paper requires angular resolution of order of $\sim$ arcmin (or less), a spectral resolution of order of $\sim 10 \mathrm{GHz}$, and a sensitivity of order of $\sim 0.1$ to $1 \%$ of the predicted SZE signals, which are achievable for integration times of minutes to hours with an experimental set up similar to the one envisaged here.

Other methods for extracting the cluster temperature profile by using SZE observations have been presented (Holder \& Loeb 2004), but they use a combination of the thermal SZE (which measures the electron pressure distribution) and radio observations of the highly polarized scattered radiation coming from active galaxies associated with the cluster (which depends on the cluster electron density distribution). Holder \& Loeb pointed out that current instruments should allow us to reach accuracies in our measurements of the mass-weighted cluster temperature profiles of order of $\sim 1 \mathrm{keV}$, but based on the assumption that the central radio source is stable over several million years. However, variable or beamed sources (such as radio galaxies or jets/lobes of AGNs associated with the cluster) will leave observable signatures in the scattered emission. Therefore, this method could allow, in principle (e.g. using $\mu \mathrm{K}$ sensitivity observations of the polarized emission), the measurement of the age of the central source by finding an edge to the polarized emission.

In this context, we note that combining this method with the independent temperature measurements from SZE spectral studies (that we discuss here) would allow us to measure the time evolution of the central radio source.

In conclusion, our study shows that SZE experiments with spectroscopic capabilities, such as those using Fourier Trasform techniques with imaging capabilities, can measure precise temperature distribution over large radial distances for galaxy clusters out to high redshift. This will allow SZE observations of clusters to be used to improve our understanding of the physics of cluster atmospheres, to reconstruct their total mass content and, eventually, to use clusters of galaxies as reliable astrophysical and cosmological probes.

\section{Appendix A: Comparing our results to the covariant formalism derivation of the SZE}

We compare the SZE calculated according to the formalism of Wright (1979; W79) with that calculated by the derivation of Bœhm \& Lavalle (2009; BL09). For the second derivation, we refer to their Eqs. (18)-(22), derived in the Thomson limit, which are therefore directly comparable to those derived by W79.

According to BL09, the correct expression of the SZE in the relativistic covariant formulation, is

$\Delta I_{\gamma}\left(E_{k}\right)=I_{\gamma}^{\text {in }}\left(E_{k}\right)-I_{\gamma}^{\text {out }}\left(E_{k}\right)$

The averages over the angles of the two contributions are given by the expressions

$\widehat{I_{\gamma}^{\text {out }}}\left(E_{k}\right)=2 \mathcal{K} \tau_{\mathrm{nr}} I_{\gamma}^{0}\left(E_{k}\right)$, where $\mathcal{K} \rightarrow 1$ in the Thomson limit, and

$$
\begin{aligned}
\widehat{I}_{\gamma}^{\mathrm{n}}\left(E_{k}\right)= & 2 \tau_{\mathrm{nr}} \int \mathrm{d} p \tilde{f}_{\mathrm{e}}\left(E_{\mathrm{p}}\right) \\
& \times \int \mathrm{d} \mu^{\prime} \int \mathrm{d} \mu \frac{\mathcal{F}\left(\beta, \mu, \mu^{\prime}\right)}{\sigma_{\mathrm{T}}} I_{\gamma}^{0}\left(t E_{k}\right) .
\end{aligned}
$$

Here, the function $\tilde{f}_{\mathrm{e}}\left(E_{\mathrm{p}}\right)$ is normalized such that $\int \mathrm{d}^{3} \boldsymbol{p} /(2 \pi)^{3} \tilde{f}_{\mathrm{e}}\left(E_{\mathrm{p}}\right)=1$, where $t=(1-\beta \mu) /\left(1-\beta \mu^{\prime}\right)$, and the function $\mathcal{F}$ is given by

$$
\begin{aligned}
\mathcal{F}\left(\beta, \mu, \mu^{\prime}\right) \equiv & \frac{\beta^{2} m^{2}}{(2 \pi)^{3}} \frac{3 \sigma_{\mathrm{T}}}{16} \frac{\left(1-\beta \mu^{\prime}\right)}{(1-\beta \mu)^{2}} \\
& \times\left\{2-2 K\left(1-\mu \mu^{\prime}\right)+K^{2}\left[\left(1-\mu \mu^{\prime}\right)^{2}\right.\right. \\
& \left.\left.+\frac{1}{2}\left(1-\mu^{2}\right)\left(1-\mu^{\prime 2}\right)\right]\right\}
\end{aligned}
$$

where $K=\left[\gamma^{2}(1-\beta \mu)\left(1-\beta \mu^{\prime}\right)\right]^{-1}$.

To quantitatively compare the two approaches, we considered an electron population with a double power-law spectrum

$f_{\mathrm{e}}(p)=A\left\{\begin{array}{cc}p^{-s_{1}} & p_{1} \leq p \leq p_{\text {break }} \\ p_{\text {break }}^{-s_{1}}\left(p / p_{\text {break }}\right)^{-s_{2}} & p>p_{\text {break }}\end{array}\right.$

where $p=\beta \gamma$, normalized to ensure that $\int_{0}^{\infty} f_{\mathrm{e}}(p) \mathrm{d} p=1$. We assumed the parameters $s_{1}=0.1, s_{2}=3.0, p_{1}=1$, and $p_{\text {break }}=100$. By using the a-dimensional momentum $p$ and the previous normalization, we note that the expression for the function $\mathcal{F}\left(\beta, \mu, \mu^{\prime}\right)$ in Eq. (A.4) slightly changes. For consistency, it must be multiplied by the factor $(2 \pi)^{3} /\left(\beta^{2} m^{2} \gamma^{2}\right)$, derived using Eqs. (16), (17), and (53) in BL09.

Figure A.1 plots the SZE spectrum over a wide frequency range, $x=(h v) /\left(k_{\mathrm{B}} T_{0}\right)$ from $x=10(v \sim 570 \mathrm{GHz})$ to $x=10^{15}$ $\left(h v \sim 2.35 \times 10^{2} \mathrm{GeV}\right)$ calculated to first order for $\tau=1$ according to W79 (continuous curve), and that calculated according to BL09 (dashed line). As can be clearly recognized, the two spectra are indistinguishable over the entire frequency range.

Figure A.2 shows a zoomed view of the SZE spectra in the microwave region $(x=0-20)$. In this region, the results obtained by the two formalisms are also indistinguishable.

Figure A.3 compares the ICS emission (against the CMB photons) along the line of sight calculated in the approach of BL09 and in the standard approach (see e.g. Colafrancesco et al. 2005; see also Longair 1993) using also the Klein-Nishina crosssection, and assuming an electron spectrum as in Eq. (A.5), with a density and spatial distribution such that $\tau=1$. The two different calculations provide quite similar results, apart for the frequency range $x>10^{15}$, at which the Klein-Nishina cross-section has a non-negligible effect (it indeed reduces the Compton scattering efficiency at high energies, see e.g. Fargion \& Salis 1998).

Based on these results, we can conclude that:

i) For the SZE spectrum in the microwave-mm region, the formalism of W79 can also be used in the case of relativistic electrons since it provides the same results as the covariant formalisms of BL09. In addition, the W79 formalism, which uses a simple expression, requires a much shorter computing time w.r.t. the BL09 formalism.

ii) When calculating the ICS emission at high frequencies, the standard formalism is completely adequate because there is 


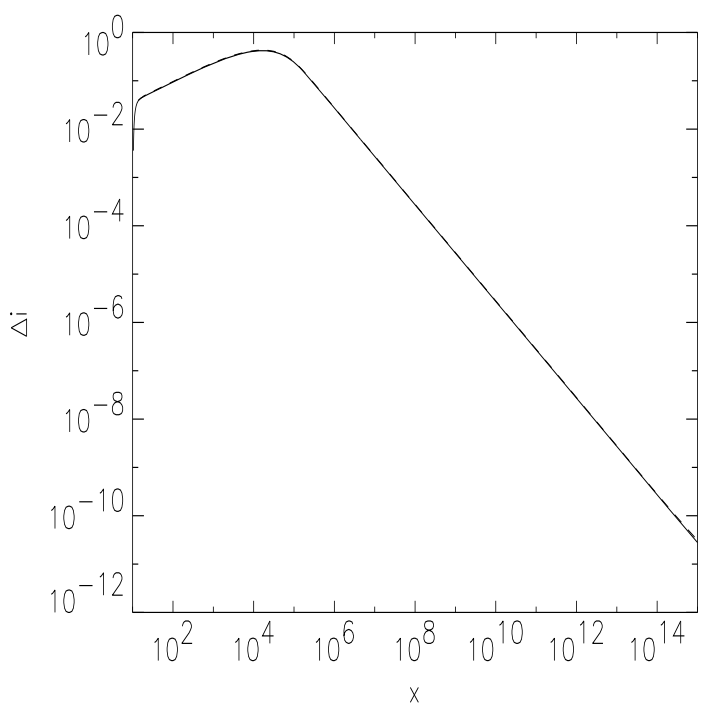

Fig. A.1. The SZE spectrum calculated following the method of W79 (solid line) and the method of BL09 (dashed line). The horizontal axis shows the adimensional frequency $x=(h v) /\left(k_{\mathrm{B}} T_{0}\right)$, while the vertical axis shows the SZE brightness change $\Delta i(x)$ in units of $2\left(k_{\mathrm{B}} T_{0}\right)^{3} /(h c)^{2}$. No difference between the two approaches is apparent.

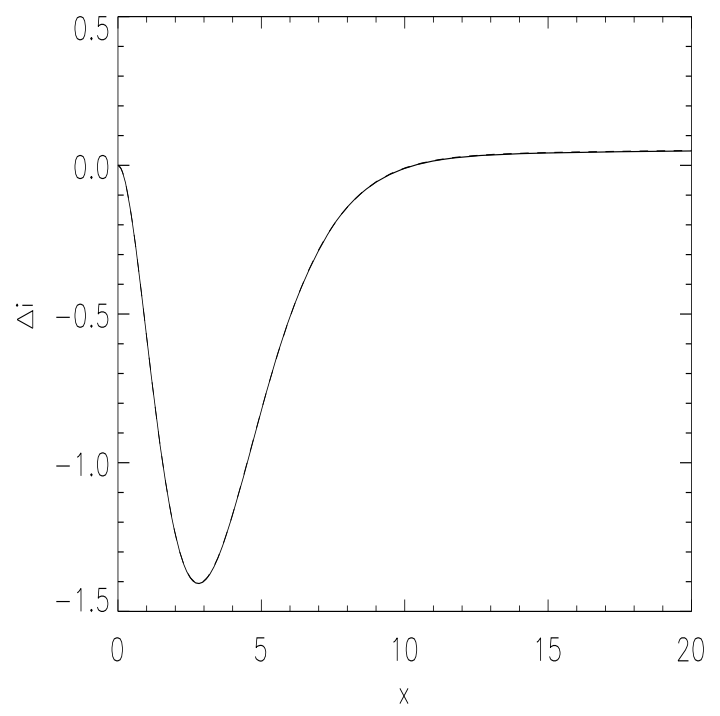

Fig. A.2. Same as Fig. A.1 but with a zoom in the microwave frequency range at which the SZE telescope operate. In this low-frequency range, there is also no difference between the two approaches.

no difference w.r.t. the covariant formalism results. In addition, the standard formalism requires a much shorter computing time and allows also the Klein-Nishina cross-section effect to be used without introducing additional complications in the numerical computation, as occurs - in contrast in Eq. (46) of BL09.

Nozawa \& Kohyama (2009) analyzed the covariant formalism of the SZE for the thermal and nonthermal distributions and derived a frequency redistribution function that is identical to that recovered by the W79 method assuming the smallness of the photon energy (in the Thomson limit). They also derived the redistribution function in the covariant formalism in the Thomson limit, and demonstrated that the two redistribution functions are mathematically equivalent in the Thomson limit, which is fully valid for the cosmic microwave background photon energies.

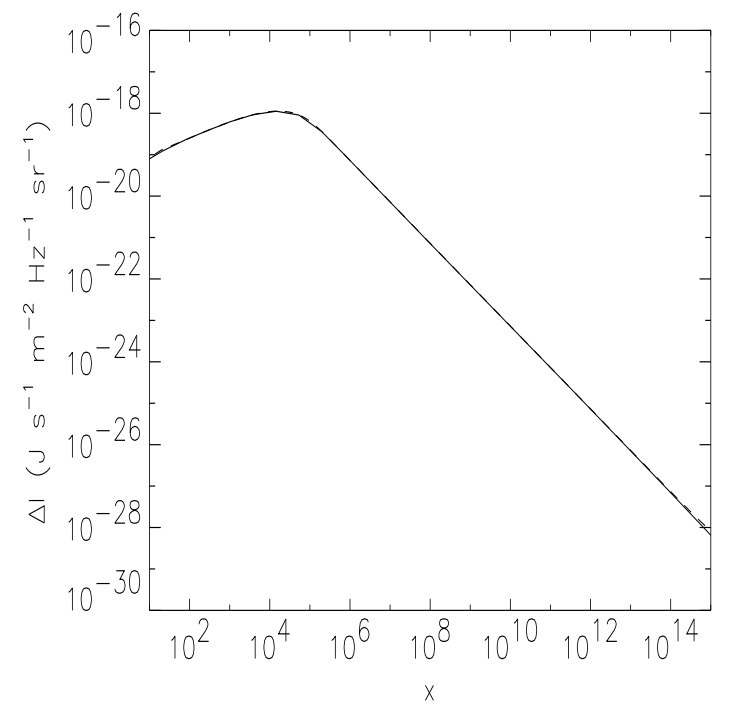

Fig. A.3. The comparison of the ICS emission evaluated following the standard computational approach with the inclusion of the KleinNishina cross-section (solid line) and that evaluated following the method of BL09 in the Thomson approximation (dashed line). No difference appears between the two approaches up to a frequency $x \sim 10^{15}$.

To summarize, the W79 and the covariant formalisms that have been developed to calculate the SZE are fully equivalent, in contrast to previous erroneous claims (see BL09), and the significant advantage of the standard calculations based on the W79 approach is their far shorter computing times.

\section{References}

Abramowitz, M., \& Stegun, I. A. 1965, Handbook of Mathematical Functions with Formulas, Graphs and Mathematical Tables (N.Y.: Dover Book) Aghanim, N., Hansen, S. H., \& Lagache, G. 2005, A\&A, 439, 901

Arnaud, M. 2005, in Background Microwave Radiation and Intracluster Cosmology, edited by F. Melchiorri, \& Y. Rephaeli, Proc. Int. School Phys. "Enrico Fermi", It. Phys. Soc. Ser., 77

Battistelli, E. S., De Petris, M., Lamagna, L., et al. 2003, ApJ, 598, L75

Birkinshaw, M. 1999, PhR, 310, 97

Bœhm, C., \& Lavalle, J. 2009, PhRvD, 79, 3505

Borgani, S., Diaferio, A., Dolag, K., \& Schindler, S. 2008, SSRv, 134, 269 Churazov, E., Forman, W., Jones, C., \& Böhringer, H. 2003, ApJ, 590, 225 Colafrancesco, S. 2004, A\&A, 422, L23

Colafrancesco, S. 2005, A\&A, 435, L9

Colafrancesco, S. 2007, New Astron. Rev., 51, 394

Colafrancesco, S. 2008a, ChJAS, 8, 61

Colafrancesco, S. 2008b, MNRAS, 385, 2041

Colafrancesco, S., \& Vittorio, N. 1994, ApJ, 422, 443

Colafrancesco, S., \& Giordano, F. 2006, A\&A, 454, L131

Colafrancesco, S., \& Giordano, F. 2007, A\&A, 466, 421

Colafrancesco, S., \& Marchegiani, P. 2008, A\&A, 484, 51

Colafrancesco, S., \& Marchegiani, P. 2009, A\&A, 502, 711

Colafrancesco, S., Mazzotta, P., Rephaeli, Y., \& Vittorio, N. 1994, ApJ, 433, 454

Colafrancesco, S., Mazzotta, P., \& Vittorio, N. 1997, ApJ, 488, 566

Colafrancesco, S., Marchegiani, P., \& Palladino, E. 2003, A\&A, 397, 27

Colafrancesco, S., Dar, A., \& De Rújula, A. 2004, A\&A, 413, 441

Colafrancesco, S., Marchegiani, P., \& Perola, G. C. 2005, A\&A, 443, 1

Colafrancesco, S., Prokhorov, D., \& Dogiel, V. 2009, A\&A, 494, 1

De Bernardis, P., Bagliani, D., Bardi, A., et al. 2010, in Proceedings of the 12th

Marcel Grossman Meeting, in press [arXiv: 1002 . 0867]

De Petris, M., D’Alba, L., Lamagna, L., et al. 2002, ApJ, 574, L119

Dolgov, A. D., Hansen, S. H., Pastor, S., \& Semikoz, D. V. 2001, ApJ, 554, 74

Ehlert, S., \& Ulmer, M. P. 2009, A\&A, 503, 35

Enßlin, T. A., \& Kaiser, C. R. 2000, A\&A, 360, 417

Ettori, S., \& Fabian, A. C. 1999, MNRAS, 305, 834

Ettori, S., Morandi, A., Tozzi, P., et al. 2009, A\&A, 501, 61

Fabian, A. C., Hu, E. M., Cowie, L. L., \& Grindlay, J. 1981, ApJ, 248, 47

Fargion, D., \& Salis, A. 1998, SvPhU, 41, 823

Finoguenov, A., Reiprich, T. H., \& Böhringer, H. 2001, A\&A, 368, 749 
A\&A 520, A31 (2010)

Guo, F., \& Oh, S. P. 2008, MNRAS, 384, 251

Halverson, N. W., Lanting, T., Ade, P. A. R., et al. 2009, ApJ, 701, 42

Hansen, S. H. 2004, MNRAS, 351, L5

Holder, G. P., \& Loeb, A. 2004, ApJ, 602, 659

Johnston, M. D., Bradt, H. V., Doxsey, R. E., et al. 1981, ApJ, 245, 799

Johnstone, R. M., Allen, S. W., Fabian, A. C., \& Sanders, J. S. 2002, MNRAS, 336, 299

Juin, J. B., Yvon, D., Réfrégier, A., \& Yèche, C. 2007, A\&A, 465, 57

Kang, H., Ryu, D., Cen, R., \& Ostriker, J. P. 2007, ApJ, 669, 729

Knox, L., Holder, G. P., \& Church, S. E. 2004, ApJ, 612, 96

Lloyd-Davies, E. J., Ponman, T. J., \& Cannon, D. B. 2000, MNRAS, 315, 689

Longair, M. 1993, High Energy Astrophysics (Cambridge University Press)

Martin, D., \& Puplett, E. 1970, Infrared Phys., 10, 105109

Mohr, J. J., Reese, E. D., Ellingson, E., Lewis, A. D., \& Evrard, A. E. 2000, ApJ, 544,109
Nozawa, S., \& Kohyama, Y. 2009, PhRvD, 79, 3005

Piffaretti, R., Jetzer, Ph., Kaastra, J. S., \& Tamura, T. 2005, A\&A, 433, 101

Pratt, G. W., Böhringer, H., Croston, J. H., et al. 2007, A\&A, 461, 71

Sato, K., Matsushita, K., \& Gastaldello, F. 2009, PASJ, 61, S365

Staniszewski, Z., Ade, P. A. R., Aird, K. A., et al. 2009, ApJ, 701, 32

Sunyaev, R. A., \& Zel'dovich, Ia. B. 1980, ARA\&A, 18, 537

Verde, L., Kamionkowski, M., Mohr, J. J., \& Benson, A. J. 2001, MNRAS, 321, L7

Voit, G. M. 2000, ApJ, 543, 113

Watanabe, M., Yamashita, K., Furuzawa, A., Kunieda, H., \& Tawara, Y. 2001, PASJ, 53605

Wright, E. L. 1979, ApJ, 232, 348

White, S. D. M., Navarro, J. F., Evrard, A. E., \& Frenk, C. S. 1993, Nature, 366, 429 